\title{
Transverse vortex-induced vibrations of a near-wall cylinder under oblique flows
}

\author{
Zhipeng Zang ${ }^{1 *}$, Tongming Zhou ${ }^{2}$

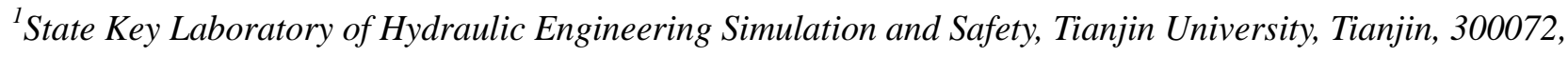 \\ China. \\ ${ }^{2}$ School of Civil, Environmental and Mining Engineering, The University of Western Australia, Crawley, WA \\ 6009, Australia. \\ *Corresponding author, Email: zangzhip@ 163.com.
}

\begin{abstract}
Transverse vortex-induced vibrations (VIVs) of a near-wall cylinder under oblique flows are studied experimentally in a water flume for both increasing and decreasing velocities. The VIV responses and the wake flow patterns around the cylinder are measured with a synchronous measurement system and a PIV. The validity of the Independence Principle (IP) for a cylinder in proximity to a wall is examined in terms of the vibration amplitude, frequency and critical normal reduced velocity. The results show that the combinations of the gap ratio and oblique angle have significant effect on the VIV response of a circular cylinder. The IP is found to be valid in predicting the VIV response for small oblique angles $\left(\theta \leq 30^{\circ}\right)$ and large gap ratios $(e / D \geq 0.8)$ whereas for large oblique angles $\left(\theta>30^{\circ}\right)$ or small gap ratios $(e / D<0.8)$, the deviations from the IP are pronounced. The upper and lower critical normal reduced velocities for the onset of VIV are obtained for both increasing and decreasing velocities. Empirical relationships between the correction factor of the critical normal reduced velocity and gap ratio and the oblique angles $\left(\theta \leq 45^{\circ}\right)$ are established. Based on the analyses of the wake flow patterns obtained by PIV, it is found that the difference in the onset of VIV for increasing and decreasing velocities corresponds to different vortex shedding modes at the initial branch: the $2 \mathrm{~S}$ vortex shedding mode for increasing velocity and the $\mathrm{C}(2 \mathrm{~S})$ mode for decreasing velocity.
\end{abstract}

Keywords: VIV; Independent Principle; oblique angle; gap ratio, critical reduced velocity. 


\section{Introduction}

When a submarine pipeline is laid on seabed, free spans may exist in many locations due to local scour or seabed unevenness. Field survey shows that the length of the free span can be more than 100 times diameter of the pipeline and most of the clearances from the seabed are within one diameter (Sumer and Fredsøe, 2006). The pipeline may undergo vibrations due to periodic vortex shedding under sea currents, which is normally termed as the vortex-induced vibration (VIV), when the shedding frequency matches the natural frequency of the structure. In practical engineering applications, VIV is crucial to the fatigue life of a spanning pipeline as the latter is closely related to the vibration amplitude and frequency (Tsahalis, 1983). Moreover, the allowable span length is usually estimated based on the critical reduced velocity to avoid VIV (DNV-RP-F105, 2006). Here, the reduced velocity is defined as $V_{\mathrm{r}}=U_{0} / D f_{\mathrm{n}}$, where $U_{0}$ is the free stream velocity, $f_{\mathrm{n}}$ is the natural frequency, $D$ is the diameter of the pipeline and the critical reduced velocity $V_{c \mathrm{r}}$ is the value of $V_{\mathrm{r}}$ for the onset of VIV. Once the critical reduced velocity is known, the allowable span length can be obtained as the natural frequency is mainly determined by the free length if other parameters are known.

VIV is a classic topic discussed by many researchers over the past decades. There are a number of comprehensive reviews on this topic, e.g. Williamson and Govardhan (2004), Sarpkaya (2004), Sumer and Fredsøe (2006) and Wu et al. (2012). Most existing studies focused on vortex shedding modes, vibration amplitude, frequency and hydrodynamic forces for a wall-free cylinder (e.g. Feng, 1968; Khalak and Williamson, 1999; Govardhan and Williamson, 2006; Klamo et al., 2006; Vandiver, 2012). However, for a near-wall cylinder (e.g. a submarine pipeline), the VIV characteristics are more complex than those of a wall-free cylinder due to the effect of the wall. 
Unlike a stationary near-wall cylinder that vortex shedding is suppressed significantly below a critical gap ratio $e / D(\approx 0.3)$, where $e$ is the gap between the cylinder surface and the wall (e.g. Lei et al., 1999; Price et al., 2002 and Wang and Tan, 2008), the elastically mounted cylinder always exhibits vortex shedding and the VIV can be observed even at a very small gap ratio, as reported by Fredsøe et al. (1987), Yang et al. (2006, 2009), Zhao and Cheng (2011) and Wang et al. (2013). Generally, with the decrease in gap ratio $e / D$, the maximum amplitude of VIV occurs at a larger reduced velocity, and both the synchronization range and the maximum upward amplitude increase; meanwhile, the dimensionless vibration frequency moves to a higher value from the Strouhal line. The vortex shedding modes for VIV of a near-wall cylinder were investigated by Zhao and Cheng (2011), Wang et al. (2013) and Zang et al. (2013). Different vortex shedding modes were identified and the effect of gap ratio on VIV responses and wake flow patterns were studied. However, the reduced velocities in these studies were constant and at relatively small values, and the maximum vibration amplitude was not reached.

In practical applications, the flows may approach the cylinder at an oblique angle $\theta$ (Fig. 1). In this study, the oblique angle $\theta$ is defined as the angle between the flow direction and the plane perpendicular to the cylinder axis. With this definition, $\theta=0^{\circ}$ refers to a flow which is perpendicular to the cylinder, or a cross-flow. The Independence Principle (IP) is commonly used in these cases to predict the hydrodynamic forces and the vibration amplitudes of the cylinder. The IP, which is also referred to as the Cosine Law, assumes that only the normal component of the free stream velocity contributes to the behaviors of the cylinder. However, previous studies have shown that the IP is usually valid when the oblique angle is smaller than a certain value. Particular attention has been paid to the validity of the IP for a stationary wall-free cylinder in estimating the 
force coefficients and the Strouhal number St (e.g. Ramberg, 1983; Zhao et al., 2009; Lam et al., 2010; Zhou et al., 2010). The Strouhal number is defined as $S t=f_{0} / U_{0} D$, where $f_{0}$ is the vortex shedding frequency. In the experimental study by Ramberg (1983) with Reynolds number $\operatorname{Re}(\equiv$ $U_{0} D / v$, where $v$ is the kinematic viscosity of the fluid) in the range of $160 \sim 1100$, the vortex shedding frequency was always larger than the value predicted by the IP for $\theta \leq 60^{\circ}$. Zhao et al. (2009) showed that the Strouhal number and the mean drag force followed the IP for $\theta \leq 60^{\circ}$ whereas the root-mean-square lift coefficients violated the IP when $\theta>30^{\circ}$ for $\operatorname{Re}=1000$, which was also observed by Lam et al. (2010). Zhou et al. (2010) observed that the secondary axial vortices were generated in the cylinder wakes and the three dimensionality of the flow was enhanced when $\theta$ was larger than $15^{\circ}$. As for a stationary near-wall cylinder, Thapa et al. (2014) showed that the IP applied to the flow at the gap ratio of 0.8 better than that at 0.4 for $R e=500$.

There are also a few efforts on the validity of the IP for VIV of a wall-free cylinder (Franzini et al., 2009, 2013; Jain and Modarres-Sadeghi, 2013; Bourguet et al., 2015). In Franzini et al. (2009), the maximum transverse vibration amplitude at $\theta=45^{\circ}$ was found lower than that at $\theta=0^{\circ}$. Their further work showed that for VIV with two degrees-of-freedom, the IP was only valid for values of $\theta$ smaller than $20^{\circ}$ (Franzini et al., 2013). In Jain and Modarres-Sadeghi (2013), the onset of VIV was observed to be approximately at the same normal reduced velocity $V_{\mathrm{rN}}\left(\equiv V_{\mathrm{r}} \cos \theta\right.$, where the subscript "N" denotes "normal") for $\theta \leq 60^{\circ}$. However, the range of the lock-in region, the maximum vibration amplitude and its corresponding normal reduced velocity failed to follow the IP entirely. Bourguet et al. (2015) numerically studied the IP for $\theta=60^{\circ}$ and found that the validity of the IP on VIV response was dependent on the axial tension of the cylinder. Till now, however, there are still no published results in the literature on the free vibration of a cylinder in considerations of 
both the oblique angle and the near-wall effects.

In this study, the VIV responses of an elastic-mounted rigid cylinder near a bottom wall under oblique flows are investigated. Firstly, to analyze parameters relevant to VIV, dimensional analysis is conducted and the experimental setup is introduced. The benchmark tests are conducted and compared with the results reported in the literature. The effects of gap ratio and cylinder oblique angle on vibration amplitude, frequency and critical normal reduced velocity $V_{\text {crN }}$ are analyzed to examine the validity of the IP. Then, a correction factor for the critical normal reduced velocities at various gap ratios and oblique angles are obtained for both increasing and decreasing velocities, and the corresponding empirical relationships are proposed further. Finally, the wake flow patterns at the initial branch of the amplitude curve for both increasing and decreasing velocities are analyzed using PIV technique and different vortex shedding modes are observed.

\section{Experimental setup and methodology}

The VIV response of a near-wall cylinder under oblique flows involves complex interactions between the flow, the cylinder and the wall. Generally, the nondimensional forms of the vibration amplitude $(A / D)$ and the frequency $\left(f / f_{n}\right)$ are the main concerned parameters. In this study, they can be determined by a group of independent parameters following the dimensional analysis as below:

$$
\begin{aligned}
& A / D=\lambda_{1}\left(e / D, \theta, V_{\mathrm{r}}, \alpha, \operatorname{Re}, \ldots\right) \\
& f / f_{\mathrm{n}}=\lambda_{2}\left(e / D, \theta, V_{\mathrm{r}}, \alpha, \operatorname{Re}, \ldots\right),
\end{aligned}
$$

where $A$ is the vibration amplitude measured from the mean position; $f$ is the vibration frequency; $e / D$ is the gap ratio with $e$ being the initial gap between the cylinder surface and the wall (Fig. 1). The combined mass-damping parameter $\alpha$ is defined as $\alpha=\left(m^{*}+C_{\mathrm{a}}\right) \zeta$, with $m^{*}$ being the mass ratio and defined as $m^{*}=4 m / \pi \rho D^{2}$, where $m$ is the total mass of the cylinder, $\rho$ is the density of the fluid; 
$C_{\mathrm{a}}$ is the potential added mass coefficient and depends on the gap ratio for a near-wall cylinder (see Section 3.1); $\zeta$ is the damping coefficient of the system. The definitions of the configuration parameters are shown in Fig. 1.

The critical reduced velocity for the onset of VIV $\left(V_{\mathrm{cr}}\right)$ is another key parameter in the analysis of VIV. In this study, it is defined as the reduced velocity $\left(V_{\mathrm{r}}\right)$ at which the value of $A / D$ equals to 0.15 at the low-velocity end of the synchronization regime, namely, $V_{\mathrm{cr}}=\left.V_{\mathrm{r}}\right|_{A / D=0.15}(\mathrm{DNV}-\mathrm{RP}-\mathrm{F} 105$, 2006; Zang and Gao, 2014). Generally, $V_{\text {cr }}$ may be influenced by a number of factors as below:

$$
V_{\mathrm{cr}}=\lambda_{3}(e / D, \theta, \Delta U, \alpha, \operatorname{Re}, \ldots)
$$

where $\Delta U$ denotes the ramp-up rate of the flow for increasing and decreasing velocities. In this study, the Reynolds number at the maximum amplitude and the mass-damping parameter $(\alpha)$ vary in a small range and their effects are not taken into account (see Govardhan and Williamson, 2006).

The experiments were conducted in the fluid-structure-soil interaction flume $(52.0 \mathrm{~m}$ long, $1.0 \mathrm{~m}$ wide and $1.5 \mathrm{~m}$ deep) at the Institute of Mechanics, Chinese Academy of Sciences. A hydro-elastic facility, which was updated from the one ever used by Zang and Gao (2014), was utilized for the physical experiments. Two sets of linear guide rails were used as the sliding devices. For more details of the VIV facility, one can refer to Zang and Gao (2014). In the present study, only transverse vibration was examined. The bottom wall of the flume was made of concrete, which can be treated as a rigid wall. The cylinder was made of plexiglass with an outer diameter $D=80$ $\mathrm{mm}$. The ends of the cylinders were cut properly so that they were parallel to the side walls of the water flume with a $5 \mathrm{~mm}$ clearance between the side wall of the flume and the end of cylinder, as shown in Fig. 1(b). Thus, the lengths of the cylinders $(L)$ for $\theta=0^{\circ}, 30^{\circ}$ and $45^{\circ}$ were $990 \mathrm{~mm}, 1143$ $\mathrm{mm}$, and $1400 \mathrm{~mm}$, which correspond to aspect ratios $L / D=12.4,14.3$ and 17.5, respectively. These 
aspect ratios are considered large enough to avoid the boundary effect of the sidewalls. The water depth in the flume was maintained at $h=0.55 \mathrm{~m}$ during the tests. The mass ratio was kept constant at $m^{*}=1.47$ for all three angles. The model pipeline was set $16.0 \mathrm{~m}$ downstream of the flow inlet. The averaged turbulence level measured in the test section was less than $3 \%$. The maximum velocity generated by the flume can be up to $0.75 \mathrm{~m} / \mathrm{s}$, which corresponds to a maximum reduced velocity $V_{\mathrm{r}} \approx 10$ for $\theta=0^{\circ}$, and the corresponding maximum Reynolds number is $R e \approx 6.0 \times 10^{4}$. The gap ratio $(e / D)$ in the tests was in the range of $0.05 \sim 1.50$.

Vibration of the cylinder was measured by a laser displacement transducer (LDT) with a sampling rate of $50 \mathrm{~Hz}$. The precision of the LDT is $\pm 1 \mathrm{~mm}$. Two Acoustic Doppler Velocimetries (ADVs) were used to measure the velocity in the flume. One was placed at $3.0 \mathrm{~m}$ upstream the cylinder to measure the free stream velocity and the other was placed at $1.5 D$ downstream to measure the wake flow velocity at the top level of the cylinder. For some cases, a LaVison PIV system with double-pulsed laser sheets upward-irradiated from the flume bottom was employed to capture the wake flow patterns of the cylinder around the onset of VIV. The experimental setup for the synchronous measurements is shown in Fig. 2. In PIV image processing, the multi-pass with decreasing window size was applied to improve the resolution and to produce less errors. The iteration was started with an initial interrogation window size of $64 \times 64$ pixels, then reduced to 32 $\times 32$ pixels with an overlapping of $50 \%$. This interrogation and evaluation procedure yielded approximately 5600 velocity vectors $(u, v)$ for each image pair. The PIV viewing area was chosen at $320 \times 240 \mathrm{~mm}($ i.e. $4.0 \times 3.0 \mathrm{D}$ ) in the $x-y$ plane, with the corresponding spatial resolution of $3.6 \times$ $3.6 \mathrm{~mm}$ (i.e. $0.045 \times 0.045 \mathrm{D}$ ). For each PIV test case, 100 pairs of flow pictures were acquired at a sampling rate of $5.03 \mathrm{~Hz}$ (i.e. $\approx 20 \mathrm{~s}$ recordings) with a time delay between the double pulses of 500 
ns. Following the procedure for the accuracy analysis due to the limitation of the number of PIV samples (Scarano and Riethmuller, 1999), the experimental uncertainty for velocity components $u$ and $v$ in this study was estimated to be $5 \%$.

The natural frequency $f_{\mathrm{n}}$ and the damping coefficient $\zeta$ were determined with the conventional free-decay test, i.e. applying a given excitation to the cylinder and then recording the vertical oscillation with the LDT. For slightly damped structures, the damping coefficient can be estimated by $\zeta=\ln \left(A_{i} / A_{i+n}\right) /(2 \pi n)$, where $A_{\mathrm{i}}$ is the initial amplitude of vibrations and $A_{\mathrm{i}+\mathrm{n}}$ is the vibration amplitude after $n$ cycles. Note that the measured damping factor includes both structural and hydrodynamic sources. In this study, the damping coefficient is $\zeta \approx 0.039$, and the mass-damping factor $(\alpha)$ is 0.098 . The natural frequency varies slightly with the gap ratio as discussed later (see Section 3.1).

The tests were conducted under both increasing and decreasing flow velocities. Brika and Laneville (1993) showed that there is a relevance between the ramp-up rate of the flow and the onset of synchronization. Prior to the tests, the effect of the ramp-up rate on the test results was evaluated first. A series of flows with ramp-up rate from $1.0 \times 10^{-4}$ to $8.0 \times 10^{-4} \mathrm{~m} / \mathrm{s}^{2}$ were applied to initiate the VIV for $e / D=0.8$. The results showed that the magnitude of $V_{\text {cr }}$ keeps almost constant for ramp-up rate less than $4.0 \times 10^{-4} \mathrm{~m} / \mathrm{s}^{2}$. Therefore, as a compromise of both flow stability and the time cost, a flow ramp-up rate of $4.0 \times 10^{-4} \mathrm{~m} / \mathrm{s}^{2}$ was used for both increasing and decreasing flow velocities in this study. After a number of repeated measurements on the onset of VIV, the uncertainty in $V_{\text {cr }}$ was found to be within $5 \%$.

The velocity profile $U(y)$ in the test section was measured from 100 pairs of flow pictures $(\approx$ $20 \mathrm{~s}$ ) using PIV for $U_{0}=0.37 \mathrm{~m} / \mathrm{s}$, as shown in Fig. 3. The velocity profile based on the logarithmic 
law for a turbulent boundary layer over a flat plate was also plotted for comparison. It can be seen that the measured velocity profile agrees favorably with the logarithmic law. The boundary layer thickness estimated with $U(y) / U_{0}=0.99$ is $\delta / D \approx 3.4$, which is very close to the estimation using the logarithmic law $(\delta / D=3.8)$. Considering that the distance from the inlet of the water flume to the test section is long enough, the boundary layer thickness at the test section will not vary significantly during the tests. Thus, for the gap ratios $(e / D=0.05 \sim 1.50)$ considered in the present study, the cylinder was still immersed in the boundary layer. The change of the boundary layer thickness due to increasing and decreasing velocities will have little effect on the results.

\section{Test results and discussions}

\subsection{Verification of the experimental setup}

To the authors' knowledge, the present study is the first attempt to evaluate the validity of IP when a vibrating cylinder is in the proximity of a solid wall. Therefore, prior to show any further results on VIV characteristics, it is important to verify the present experimental setup by comparing the measured results at simpler setup with those reported previously. To quantify the reduced velocity, the natural frequency of the cylinder close to a wall should be determined first. These values are obtained through free decay tests and the results are shown in Fig. 4 for different oblique angles. Values of $f_{\mathrm{n}}$ estimated with the theoretical method by Fredsøe et al. (1987), via.

$$
f_{n}=\frac{1}{\pi D} \sqrt{\frac{k}{\pi \rho\left(C_{a}+m^{*}\right)}},
$$

are also shown for comparison. In Eq. (4), $k$ is the spring coefficient per unit span of the cylinder; $C_{\mathrm{a}}$ is the added mass coefficient and taken from Yamamoto et al. (1974). Generally, the values of $C_{\mathrm{a}}$ decrease with the increase of $e / D$ and reaches a constant value of 1.0 for an isolated one (i.e. $e / D \approx$ 
$\infty)$. It is seen from Fig. 4 that the theoretical values of the natural frequency agree well with the measured ones for the three oblique angles. They decrease slightly with the decrease of the gap ratio due to the increase in the added mass as the cylinder approaches the wall. In this study, the frequency for small gap ratios $(e / D<0.25)$ cannot be obtained from the free-decay tests due to the constraint of the bottom wall; instead, the theoretical values calculated by Eq. (4) are used.

Fig. 5 shows the vertical displacement of the cylinder in a cross flow (i.e. $\theta=0^{\circ}$ ) for $e / D=$ $1.25,0.25$ and 0.05 under both increasing and decreasing velocities. For $e / D=1.25$, there is almost no influence from the bottom wall and the vibration displacements are symmetric about the initial equilibrium position. The critical reduced velocities for the onset of VIV for both increasing and decreasing velocities are very close $\left(V_{\mathrm{r}} \approx 3.5\right)$ (Please note: they will be defined as the upper and lower critical normal reduced velocities $V_{\mathrm{crN}}{ }^{+}$and $V_{\mathrm{crN}}{ }^{-}$later in Figure 15). With the decrease of the gap ratio $e / D$ to 0.25 and 0.05 , the transverse vibration is not as symmetric as that for the wall-free case. The impact between the cylinder and the bottom wall was observed in the test, which can also be reflected by the fact that the maximum downward displacements (towards the bottom) equal to the gap ratio. The critical reduced velocity of VIV for increasing velocity is larger than that for decreasing velocity and the difference between them becomes larger with the decrease of the gap ratio. This difference is expected to be related with the flow patterns at the onset of VIV for increasing and decreasing velocities, as will be discussed in Sec. 3.5. Fig. 6 shows the vertical displacement of the cylinder with $\theta=45^{\circ}$ for $e / D=0.80,0.25$ and 0.05 . Generally, the displacement curves for $\theta=45^{\circ}$ are similar to those for $\theta=0^{\circ}$, but the values of $V_{\mathrm{crN}}$ of the former are larger than those of the latter, and the vibration amplitudes of the former are slightly smaller than those of the latter. 
Because there are no results available in the literature for VIV of a circular cylinder in considerations of both the gap ratio to a wall and the oblique angle to the flow, the present results are compared with those of near-wall cylinders in a cross flow and with those of wall-free cylinders under oblique flows, separately. In evaluating the vibration amplitude $A$ for a given reduced velocity, 20 vibration amplitudes around this particular instant/velocity were averaged. Fig. 7 shows the variations of $A / D$ and $f / f_{\mathrm{n}}$ with $V_{\mathrm{r}}$ for a near-wall cylinder in a cross flow $\left(\theta=0^{\circ}\right)$. The results obtained by Yang et al. (2009) at similar gap ratios are also included for comparison. The mass-damping factor in the present study is $\alpha=0.098$ which is close to $\alpha=0.075$ used in Yang et al. (2009). In both studies, the bottoms are made of rigid concrete wall, which makes the interactions between the bottom and the cylinder comparable. For $e / D=0.63$ and $0.80, A / D$ reaches the maximum values quickly, and then decrease with the reduced velocity. For $e / D=0.05, A / D$ increases gradually with the reduced velocity, and yet to achieve the maximum value in the present tests, owing to the flow speed limitation. The present results of $A / D$ are slightly larger than those reported by Yang et al. (2009), which could be because of the difference in Reynolds numbers corresponding to the maximum values of $A / D$ in the two studies. The value of $R e$ at the maximum of $A / D$ is $6.0 \times 10^{4}$ in the present study whereas that in Yang et al. (2009) is about $1.9 \times 10^{4}$. Govardhan and Williamson (2006) have shown that the maximum vibration amplitude increases with increasing Reynolds number for a given damping-mass factor. Another reason could be the difference in the boundary layer thickness and the relative location of the cylinders in the boundary layers between the two studies. The boundary layer thickness $\delta$ in this study is about $3.4 \mathrm{D}$ but it was not given in Yang et al. (2009). In this study, the vibration frequency was obtained through FFT. The variations of $f / f_{\mathrm{n}}$ with $V_{\mathrm{r}}$ also share the same trends in both the present study and that in Yang et 
al. (2009) as shown in Fig. 7(b). The overall agreement between these two studies is acceptable.

Fig. 8 compares the values of $A / D$ and $f / f_{\mathrm{n}}$ for a wall-free cylinder $(e / D \approx \infty)$ at different oblique angles with those reported by Franzini et al. (2013). It can be seen that the maximum value of $A / D$ in the present study is about 5\% smaller than that in Franzini et al. (2013) for all angles. This difference may be caused by the difference in the mass-damping factor $\alpha$ between the two studies. It is 0.098 in the present study which is larger than that in Franzini et al. (2013) $(\alpha=0.0026)$. It has been shown previously (e.g. Govardhan and Williamson, 2006; Zhou et al., 2011) that the maximum vibration amplitude generally decreases with increasing mass-damping factor. The variations of $f / f_{n}$ with $V_{\mathrm{rN}}$ in the two studies also show the same trend. The results in Figs. 7 and 8 confirm a satisfactory verification to our experimental setup.

\subsection{Effect of gap ratio on VIV responses}

In this section, the effect of gap ratio on VIV responses is assessed based on the values of $A / D$, $f / f_{\mathrm{n}}$ and $V_{\text {cr. }}$. Owing to the limitation of the flow speed generated by the flume, the maximum value of $V_{\text {cr }}$ can only reach 10 for $\theta=0^{\circ}$. Thus, the full range of the synchronization region could not be covered, especially for small gap ratios and large oblique angles.

Fig. 9 shows the variations of $A / D$ with $V_{\mathrm{r}}$ for various values of $e / D$ at three different oblique angles for both increasing and decreasing flow velocities. It is seen that the increase of $A / D$ from nearly zero to the maximum values depends apparently on $e / D$. $A / D$ increases more rapidly to the maximum value for a larger $e / D$ than that for a smaller $e / D$, i.e., with the decrease of $e / D$, the maximum value of $A / D$ occurs at a higher value of $V_{\mathrm{rN}}$. For example, when $e / D=0.8$ and $\theta=0^{\circ}$ (see Figs. 9a and 9b), $A / D$ achieves the maximum value at $V_{\mathrm{r}} \approx 4.5$ with a jump from zero, whereas when $e / D=0.05, A / D$ increases gradually and even at $V_{\mathrm{r}}=10$, the maximum value hasn't been 
achieved. This phenomenon was also observed by Fredsøe et al. (1987) and Raghavan et al. (2009). Raghavan et al. (2009) attributed this to the interactions between the boundary layer of the plane wall and the shear layer emanating from the lower side of the cylinder. Thus, vortex shedding at larger gap ratios is much stronger than that at smaller gap ratios. This can be clearly seen from the vortex shedding strength for a cylinder by the PIV results shown later. The same trend is also applicable to the cases for $\theta=30^{\circ}$ and $45^{\circ}$ (Figs. 9c $\sim 9$ f). The maximum amplitude also occurs at a higher reduced velocity with the increase of the oblique angle.

In Fig. 9, the critical reduced velocity $\left(V_{\text {cr }}\right)$ for the onset of VIV decreases with the increase of $e / D$. For example, when $\theta=0^{\circ}, V_{\text {cr }}$ is about 5.0 for $e / D=0.05$, and it decreases to about 3.0 for $e / D$ $=0.80$. This phenomenon can be related with the lift and vortex shedding characteristics for different gap ratios. As the gap ratio is large enough (e.g. $e / D \geq 0.8$ ), the influence from the wall is negligible and vortex shedding and the resulting lift force are strong. When $e / D$ is small, vortex shedding is suppressed and the lift force is also small. The value of $V_{\mathrm{cr}}$ for decreasing flow velocity are almost in the range of $2.0 \sim 3.0$, smaller than their corresponding values for the increasing flow velocity. The same trend also occurs for the cases with oblique angles of $\theta=30^{\circ}$ and $45^{\circ}$. This can be explained by that when the reduced velocity approaches the critical value for decreasing velocity, due to the disturbance to the flow caused by vortex shedding, the cylinder will keep vibrating till a lower value than the critical reduced velocity for increasing velocity. The quantitative analysis on the effects of the gap ratio and oblique angle on the critical reduced velocity will be conducted in following sections.

Fig. 10 shows the variations of $f l f_{\mathrm{n}}$ with $V_{\mathrm{r}}$ for different values of $e / D$ at three oblique angles. In the figures, the hollow symbols denote results for decreasing velocity while the solid symbols are 
for both increasing and decreasing velocities. Two auxiliary lines for the normalized vortex shedding frequency of a stationary wall-free cylinder (Strouhal line), $f / f_{\mathrm{n}}=0.2 V_{\mathrm{r}}$, and the natural frequency, $f / f_{\mathrm{n}}=1.0$, are also shown in the figure for reference. The values of $f / f_{\mathrm{n}}$ increase with the increase of $V_{\mathrm{r}}$, and becomes higher with the decrease of $e / D$. This trend is in agreement with that reported by Fredsøe et al. (1987), Yang et al. (2009), Raghavan et al. (2009) and Wang et al. (2013) for $\theta=0^{\circ}$. Fredsøe et al. (1987) explained this partly by chopping of the downward displacement by the bottom and partly by the self-excited vibration due to the transfer of energy from the flow to the cylinder. They showed a combination of VIV and wall-induced oscillations due to the asymmetric wake dynamics. Raghavan et al. (2009) attributed the higher frequency to (i) chopping of the downward displacement by the bottom, and (ii) stronger vortex structures. The presence of a wall in proximity of the cylinder exerts complicated effects on the cylinder response in terms of vibration amplitude and frequency modulation. Based on the FFT of PIV results, it is shown that the vortex shedding frequency from a vibrating cylinder increases with decreasing gap ratio, and is seen to synchronize with the vibration frequency (Wang et al., 2013).

To examine the dependence of $A / D$ on $e / D$ more clearly, the value of $A / D$ at $V_{\mathrm{rN}}=5.1$ are plotted against $e / D$ for the three oblique angles and compared with those reported by Wang et al. (2013) for $\theta=0^{\circ}$, as shown in Fig. 11. It is seen that $A / D$ increases with the increase of $e / D$ and approaches an asymptotic value of 0.8 when $e / D=1.25$. It is also seen that $A / D$ decreases with the increase in $\theta$ and the discrepancies among the three oblique angles for small values of $e / D$ are more significant than those for large values of $e / D$. This result should be related with the special flow characteristics at large oblique angles, at which, the secondary or streamwise vortices have been enhanced at the expense of the primary spanwise vortices, resulting a reduction of the large-scale 
spanwise vortical structures and hence a less pronounced force-displacement synchronization process (Zhou et al., 2010; Franzini et al., 2013).

\subsection{Effect of oblique angle}

The variations of $A / D$ and $f / f_{\mathrm{n}}$ with $V_{\mathrm{rN}}$ at several gap ratios are shown in Figs. 12 and 13 to examine the validity of the IP for a near-wall cylinder. If the IP is valid, the vibration amplitude and frequency will approximately collapse on a unique curve for different oblique angles. In Figs. 12(a) and $12(\mathrm{~b})$, for $e / D=0.8$, there is satisfactory agreement of $A / D$ among different oblique angles for both increasing and decreasing velocities, with a deviation less than $5 \%$ in the maximum amplitude and critical normal reduced velocity between $\theta=0^{\circ}$ and $30^{\circ}$. These results indicate that the IP is valid for small oblique angles $\left(\theta \leq 30^{\circ}\right)$ at large gap ratios (Franzini et al., 2013; Thapa et al., 2014). In Figs. $12(\mathrm{c}) \sim 12(\mathrm{f})$, for $e / D=0.38$ and 0.05 , the differences in the vibration amplitude and the critical normal reduced velocity among the three oblique angles are apparent and the maximum vibration amplitude decreases with the increase of $\theta$. The decrease in the maximum amplitude with the increase of $\theta$ is coincident with the findings by Lucor and Karniadakis (2003), Franzini et al. (2009, 2013), Jain and Modarres-Sadeghi (2013), etc. This can be explained by the fact that the increase in $\theta$ leads to the presence of the axial flow, which may not only deteriorate the spanwise flow structures and enhance the three-dimensionality of the flow, but also modulate the phase shift between the force and displacement, resulting in a less pronounced force-displacement synchronization process (Franzini et al., 2013). The larger the phase modulation is, the larger the decrease in the oscillation amplitude is. This result indicates that the IP is not valid for small values of $e / D$ even though the oblique angle is small. Furthermore, due to the suppression of vortex shedding with the decrease of the gap ratio, the force-displacement synchronization process is 
further decreased, which makes the differences among the three oblique angles for $e / D=0.38$ and 0.05 more pronounced than those for $e / D=0.8$. The critical normal reduced velocity is also very dependent on the oblique angle and will be discussed in Section 3.4.

Fig. 13 shows the variations of $f / f_{\mathrm{n}}$ with $V_{\mathrm{rN}}$ for the three oblique angles. $f / f_{\mathrm{n}}$ increases with the increase of $V_{\mathrm{rN}}$ for all cases. For $e / D=0.8$, the values of $f / f_{\mathrm{n}}$ for the three oblique angles agree well with each other, whereas with the decrease of $e / D$ to 0.38 (Fig. 13b) and 0.05 (Fig. 13c), the discrepancies among the three oblique angles are pronounced. To reveal the trend of $f / f_{\mathrm{n}}$ with $e / D$ more clearly, Fig. 14 shows the value of $f / f_{\mathrm{n}}$ with $e / D$ at $V_{\mathrm{rN}}=5.1$ for the three oblique angles. $f / f_{\mathrm{n}}$ decreases with the increase of $e / D$ when the latter is smaller than about 0.8 , and then approach a constant value of around $f / f_{\mathrm{n}}=1.0$ for large values of $e / D$. The differences in the values of $f / f_{\mathrm{n}}$ among different angles are small when $e / D \geq 0.8$. However, the differences of $f / f_{\mathrm{n}}$ for the three oblique angles are getting pronounced with the decrease of $e / D$ when $e / D$ is smaller than 0.8 . For example, when $e / D=0.05$, the value of $f / f_{\mathrm{n}}$ at $\theta=45^{\circ}$ is about 1.5 times of that at $\theta=0^{\circ}$. The 3D vortex shedding flows by Thapa et al. (2014) showed that with the increase of $\theta$ and the decrease of $e / D$, the three-dimensionality of the flow becomes weaker, which corresponds to the strong spanwise vortices and weak streamwise vortices. Furthermore, the spanwise flow becomes stronger with the increase of $\theta$ for a given normal reduced velocity. The strong spanwise vortices results in an increase of the lift fluctuation and frequency (Thapa et al., 2014).

From the above discussion, it can be seen that the proximity of a cylinder to a wall can affect the validity of the IP. When $e / D$ is larger than 0.8 , the vibration amplitude and frequency collapse well on a single value, and they scatter apparently when $e / D$ is smaller than 0.8 , especially for $\theta=$ $45^{\circ}$. These results indicate that the IP is valid in predicting the VIV responses at small oblique 
angles $\left(\theta \leq 30^{\circ}\right)$ and large gap ratios $(e / D \geq 0.8)$. With the decrease of $e / D$, the deviation from the IP becomes large, even when the oblique angle is small. The IP neglects the effect of the axial flow component, which seems reasonable for small oblique angles but not for the large ones due to the strong influence from the axial flow. The above result seems consistent with the conclusions by Thapa et al. (2014) that the IP was satisfied better at $e / D=0.8$ than at $e / D=0.4$, and the force coefficients for $e / D=0.4$ do not follow the independence principle if $\theta$ is greater than $30^{\circ}$ for a stationary cylinder at $R e=500$.

\subsection{Critical reduced velocities of VIV}

To examine the effect of increasing and decreasing flow velocity on VIV responses, Fig. 15 shows the variations of $A / D$ with $V_{\mathrm{rN}}$ for various values of $e / D$ and $\theta$. The difference for the onset of VIV at the initial branch of the amplitude curve between the increasing and decreasing velocities can be seen clearly. This difference exists for all cases in the present study and depends apparently on $e / D$ and $\theta$ and becomes more pronounced with the decrease of $e / D$ or the increase of $\theta$. However, for the lower branch of the amplitude curve, increasing or decreasing velocity does not influence the VIV response of the cylinder.

The difference for the onset of VIV under increasing and decreasing velocities can be quantified by the upper and lower critical normal reduced velocities, defined as $V_{\mathrm{crN}}{ }^{+}$and $V_{\mathrm{crN}}{ }^{-}$, in Fig. 15(c). There were a number of studies on the VIV of a cylinder near a plane boundary, in which the critical reduced velocity varies significantly due to the differences in flow and structural parameters. In DNV-RP-F105 (2006), a correction factor $\psi$ was introduced to consider the effect of the proximity to the seabed on the critical reduced velocity, excluding the effects of other parameters. In this study, the correction factor $\psi$ is also applied to investigate the effects of the gap 
ratio and oblique angle on the critical normal reduced velocity. The correction factor $\psi$ is defined as:

$$
\psi=\frac{V_{\mathrm{crN}}}{V_{\mathrm{crN} 0}}
$$

where $V_{\mathrm{crN}}\left(\equiv V_{\mathrm{cr}} \cos \theta\right)$ denotes the critical normal reduced velocity in considerations of $e / D$ and $\theta$ for both increasing and decreasing velocities; $V_{\mathrm{crN} 0}$ denotes the critical reduced velocity for a wall-free cylinder in a cross flow, i.e. $\theta=0^{\circ}$ and $e / D \approx \infty$.

Fig.16 shows the variations of $\psi$ for both increasing and decreasing velocities with $e / D$ for the three oblique angles. For $\theta=0^{\circ}$ (Fig. 16a), the existing results by Fredsøe et al. (1987), Yang et al. (2006, 2009) and Tham et al. (2015) are also plotted for comparison. For increasing velocity, all available results of $\psi$ decreases with the increase of $e / D$ when $e / D \leq 0.8$, which indicates that the onset of VIV occurs at a larger normal reduced velocity for smaller $e / D$. For decreasing velocity, the results of $\psi$ by the present tests agree well with those by Fredsøe et al. (1987), which are obtained by forcing the pipe to vibrate when the velocity is small. Although the methods in the two studies are different, they both represent the onset of VIV with external disturbance. Contrary to that for increasing velocity, the values of $\psi$ for decreasing velocity increase with the increase of $e / D$ when $e / D \leq 0.8$, indicating that VIV can persist even at a small reduced velocity for small gap ratios. This may be explained by the fact that due to the disturbance to the flow caused by stronger vortex shedding compared with a stationary cylinder, the cylinder will keep vibrating till a lower value of reduced velocity for decreasing velocity than that for increasing velocity. When $e / D>0.8$, the values of $\psi$ for both increasing and decreasing velocities vary slightly with a discrepancy being less than $5 \%$. It can also be seen that the values of $\psi$ for increasing velocity are always larger than that for decreasing velocity even for large values of $e / D$. 
The variations of $\psi$ with $e / D$ for $\theta=30^{\circ}$ and $45^{\circ}$ (Figs. 16b and c) almost have the same trend as that for $\theta=0^{\circ}$. For $e / D \leq 0.8$, the values of $\psi$ for increasing velocity decrease with the increase of $e / D$ and those of $\psi$ for decreasing velocity increase with the increase of $e / D$. For $e / D>0.8$, the values of $\psi$ both for decreasing and increasing velocities approach a constant value. The experimental results of $\psi$ for a wall free cylinder by Franzini et al. (2013) and Jain and Modarres-Sadeghi (2013) fit the present trend well.

It is pertinent to propose a general formula for $\psi$ which can account for the effects of both the gap ratio and the oblique angle. In this study, the formula is obtained by fitting the experimental results and expressed as below (for $\theta \leq 45^{\circ}$ ):

$$
\psi=\frac{0.3}{\cos \theta} \operatorname{ex~}^{A_{1}\left(0-\frac{8}{D}\right.}+A_{2} \frac{e}{D} \quad(e / D \leq 0.8)
$$

in which, the coefficients $A_{1}$ and $A_{2}$ are taken as:

$$
\begin{array}{ll}
\text { for increasing velocity: } & A_{1}=1.95, A_{2}=0.88 \\
\text { for decreasing velocity: } & A_{1}=0.95, A_{2}=0.82 .
\end{array}
$$

When $e / D>0.8$, the near-wall effect becomes negligible and Eq. (7) can be simplified as:

$$
\psi=\frac{0.3}{\cos \theta}+0.88_{2} \quad(e / D>0.8)
$$

The predictions of $\psi$ using Eqs. (7) \& (8) are also included in Fig. 16 for comparison with the existing results. It is seen that they can represent the existing results well for all cylinder oblique angles. Furthermore, a correlation analysis was conducted between the experimental results and the predicted values as shown in Fig. 17. Two auxiliary lines with $\pm 15 \%$ tolerance are plotted for reference. It is seen that most of the data points are located between the two auxiliary lines and the deviations between them are within $15 \%$ for most of the cases. 
A design plot for $\psi$ varying with $e / D$ for $\theta \leq 45^{\circ}$ is shown in Fig. 18. The values of $\psi$ given by DNV-RP-F105 (2006) are also plotted in the figure. There is a unique curve for $\psi$ by DNV-RP-F105 (2006) because the IP is assumed to be tenable for all angles and the difference between increasing and decreasing velocities is not considered as well. However, the present study shows that the values of $\psi$ depend apparently not only on the cylinder oblique angle but also on the increasing or decreasing velocity. Therefore, the increasing and decreasing trends of $V_{\mathrm{rN}}$ on $\psi$ should be considered in the determination of $\psi$. It is seen from Fig. 18 that the values of $\psi$ given by DNV-RP-F105 (2006) agree very well with the values of $\psi$ at $\theta=0^{\circ}$ and $30^{\circ}$ for the lower critical normal reduced velocity $\left(V_{\mathrm{crN}}{ }^{-}\right)$. It is indicated that the values of $\psi$ by DNV-RP-F105 (2006) may be taken from decreasing velocities or forced vibrations at small velocities as those adopted in Fredsøe et al. (1987). Thus, the predicted values of $\psi$ by the DNV code are in the most conservative situation. It also suggests that IP is reasonable to predict the critical normal reduced velocity for $\theta \leq$ $30^{\circ}$ as the difference between the values of $\psi$ for $\theta=0^{\circ}$ and $30^{\circ}$ is less than $15 \%$. However, for $\theta=$ $45^{\circ}$, the difference between values of $\psi$ for $\theta=0^{\circ}$ and $45^{\circ}$ is more than $40 \%$, indicating that the IP is not valid anymore for $\theta=45^{\circ}$. Although the lower critical normal reduced velocity is for the case of VIVs under a decreasing velocity, it is essentially for the onset of VIV with the external disturbances. The upper critical normal reduced velocity $\left(V_{\mathrm{crN}}{ }^{+}\right)$is for the onset of VIV without any disturbance. Fig. 18 provides more options than DNV-RP-F105 (2006) for predicting the onset of VIV under various flow conditions.

\subsection{Vortex shedding modes}

As discussed above, the critical reduced velocities are different for increasing and decreasing flow velocities, which correspond to the different initial branches (see Fig. 15). In this section, the 
flow patterns around the cylinder at the initial branches for both increasing and decreasing velocities at $V_{\mathrm{r}}=4.0$ for $\theta=0^{\circ}$ are examined using PIV to further explore the underlying physics of the differences for the onset of VIV. The flow fields around a wall-free stationary cylinder at the same reduced velocity are also shown for comparison.

When a cylinder is placed close to a solid boundary below a critical $e / D$, changes in the flows around the cylinder will take place, such as the break-up of the symmetry and the suppression of vortex shedding. The suppression of vortex shedding is closely linked with the asymmetry in the development of the vortices on both sides of the cylinder because the shear layer on the wall-side will not develop as strongly as that on the free-stream side. This can be reflected by the time-averaged swirling strength fields of the wake flow. Here, the swirling strength $\Omega$ is defined as:

$$
\Omega=\max (0,-\Delta / 4)
$$

where $\Delta$ is the discriminant of the characteristic equation of the $2 \mathrm{D}$ velocity gradient tensor, and can be expressed as (Chong et al., 1990):

$$
\Delta=\left(\frac{\partial u}{\partial x}+\frac{\partial v}{\partial y}\right)^{2} 4\left(\frac{\partial u \partial v}{\partial x \partial y}-\frac{\partial u}{\partial y} \hat{o}\right.
$$

The swirling strength can discriminate the vortices shed from the cylinder and exclude the shear motion generated by the wall boundary. It has been used previously to study the vortex shedding from cylinders near a plane boundary (e.g. Chong et al., 1990; Zhou et al., 1999; Zang et al., 2013). Fig. 19 shows the contours of the swirling strength for a stationary cylinder in a cross-flow $\left(\theta=0^{\circ}\right)$ at $\operatorname{Re}=2.4 \times 10^{4}$ (or equivalent to $\left.V_{\mathrm{r}}=4\right)$ for both wall-free $(e / D \approx \infty)$ and near-wall $(e / D=0.63$ and 0.25 ) conditions. It can be seen that for the wall-free cylinder, the swirling strength field is perfectly symmetry about the centerline of the cylinder. With the decrease of $e / D$, vortex shedding has been suppressed on both sides compared with that for a wall-free case. When the gap ratio is reduced to 
$e / D=0.25$, the asymmetry of vortex shedding on both sides becomes more apparent and the vortex strength on the wall-side of the cylinder decreases faster (by $\approx 70 \%$ ) than that on the free-stream side (by $\approx 35 \%$ ), indicating partial or complete suppression of the regular vortex shedding due to the plane wall.

Fig. 20 shows typical snapshots of the velocity vectors for a stationary and a vibrating cylinder in a cross-flow $\left(\theta=0^{\circ}\right)$ at gap ratios $e / D=0.63$ (left column) and 0.25 (right column). For the stationary cylinder with $e / D=0.63$ (Figs. 20a and 20b), it is seen that the alternate vortex shedding takes place downstream of the cylinder. The vortex shedding pattern is in $2 \mathrm{~S}$ mode, which can be identified by two single vortices of opposite sign shed from the cylinder in each vibrating period. This result indicates that the suppression of vortex shedding by the bottom wall is not significant. For $e / D=0.25$, there is only one clockwise vortex shed from the upper shear layer of the cylinder while the vortex shedding at the bottom of the cylinder is inhibited by the wall because this gap ratio is smaller than the critical value of about 0.3 (e.g. Lei et al., 1999; Price et al., 2002; Wang and Tan, 2008).

For the case of the vibrating cylinder, however, the vortex shedding patterns are dictated by both the increasing/decreasing flow velocity and the gap ratio. Figs. 20(c-f) shows snapshots of the velocity vectors at gap ratios $e / D=0.63$ and 0.25 both for increasing and decreasing velocities. The instantaneous positions of the cylinder are also illustrated in Fig. 20, in which $y_{\max }$ and $y_{\min }$ denote the upper and lower boundaries of the vibration displacement, respectively; $C$ denotes the center of the pipeline. For the flow field around the vibrating cylinder with increasing velocity at $e / D=0.63$ (Fig. 20c), it is seen that the wake flow pattern is still in $2 \mathrm{~S}$ mode. But the shed vortices are more regular and the vortex-street becomes wider compared with those of the stationary cylinder. It 
seems that the vibration of the cylinder increase the vortex strength, resulting in vortex shedding to be more regular and more organized as compared with that of a stationary cylinder at the same gap ratios. For decreasing velocity shown in Fig. 20(e), there is still alternate vortex shedding behind the cylinder, but the two single vortices develop into two parallel rows and coalesce in the far wake, which is referred to as the $\mathrm{C}(2 \mathrm{~S})$ mode. Although the reduced velocity for the flow fields in Figs. 20(c) and 20(e) are the same, the vortex shedding modes are different from each other. This may be explained by the fact that the vortex shedding modes are dependent on the vibration amplitude as the amplitude for increasing velocity is higher than that for decreasing velocity at the initial branch. This result supports our argument about the difference in critical reduced velocity for increasing and decreasing velocities (e.g. Figs. 15 and 16). Tham et al. (2015) also found that the $\mathrm{C}(2 \mathrm{~S})$ mode occurs for high-amplitude vibrations whereas the $2 \mathrm{~S}$ mode occurs for low-amplitude vibration at the initial branch for a vibrating cylinder near a plane wall.

Fig. 20(d) shows the flow field of the vibrating cylinder at $e / D=0.25$ for increasing velocity. For this gap ratio, the impact between the cylinder and the bottom takes place due to constraint effect of the latter. Different from the vortex suppression by the bottom for a stationary cylinder, regular vortex shedding can be observed behind the vibrating one although the positive vortices from the bottom of the cylinder are much weaker than the negative vortices from the top. Zhao and Cheng (2011) classified the wake flow pattern of a vibrating cylinder with impact from a bottom wall into three vortex shedding modes, namely, "single vortex mode", "vortex-shedding -after-bounce-back mode" and "vortex-shedding- before-bounce-back mode", depending on the reduced velocity. In this case, the vortex shedding mode may belong to the "vortex-shedding-after-bounce-back mode". In Fig. 20(f), for decreasing velocity at $e / D=0.25$, 
there are also two rows of vortices, but one row of vortices is heavily cancelled due to the interactions with the bottom. This can also be considered as a type of $\mathrm{C}(2 \mathrm{~S})$ mode. Wang et al. (2013) and Tham et al. (2015) also observed that when $e / D$ is relatively small, vortex shedding occurs only in the upper shear layer, forming a similar vortex street.

In this study, the differences for the onset of VIV for both increasing and decreasing velocities may indicate a hysteresis effect for the onset of VIV, as reported by Singh and Mittal (2005) and Prasanth et al. (2011). In these studies, the hysteresis was also associated with the switch between the $2 \mathrm{~S}$ and the $\mathrm{C}(2 \mathrm{~S})$ modes of vortex shedding for the onset of synchronization. However, the confirmation of the hysteresis effect may need precise measurements of the phase angle between the forces and the cylinder displacement, which are absent in the present study and are worth to be conducted in the future.

\section{Conclusions}

VIV responses of a near-wall cylinder under oblique flows are experimentally investigated for both increasing and decreasing velocities. The vibration amplitude, frequency and the critical reduced velocity are quantitatively studied to evaluate the validity of the Independent Principle (IP) in the near wall region. The experimental setup of the present study is validated first. Then, the effects of gap ratio and oblique angle on VIV characteristics are discussed. Differences at the onset of VIV were found for increasing and decreasing velocities. To further examine the underlying physics related with the above differences in critical reduced velocities, velocity vector plots captured with PIV are analyzed. The main conclusions are summarized as follows:

(1) The combinations of the gap ratio and the oblique angle have significant effect on the VIV 
responses of a near-wall circular cylinder. The IP is valid in predicting the amplitude, frequency and critical reduced velocity of VIV for small oblique angles $\left(\theta \leq 30^{\circ}\right)$ and large gap ratios $(e / D \geq 0.8)$ whereas for small gap ratios $(e / D<0.8)$ or large oblique angles $\left(\theta>30^{\circ}\right)$ the deviations from the IP are pronounced.

(2) The upper and lower critical normal reduced velocities for the onset of VIV are obtained for both increasing and decreasing velocities. A correction factor accounting for the effects of gap ratio and oblique angle is formulated by fitting the experimental results, which can well supplement the results reported in DNV-RP-F105 (2006) for predicating the onset of VIV.

(3) Based on the analyses of the wake flow patterns with PIV, it is found that the differences for the onset of VIV with increasing and decreasing velocities correspond to different vortex shedding modes at the initial branch: the $2 \mathrm{~S}$ vortex shedding mode for the increasing velocity and the $\mathrm{C}(2 \mathrm{~S})$ mode for the decreasing velocity.

\section{Acknowledgements}

This research was supported by the General Programs (Grant nos. 51109202, 51579232), the Science Fund for Creative Research Groups (Grant no 51021004) of National Natural Science Foundation of China and the Open Funding of State Key Laboratory of Coastal and Offshore Engineering (LP1410). T. Zhou sincerely acknowledges the financial supports from Australian Research Council through ARC Discovery Projects DP110105171 and DP130104535. The authors would also like to appreciate the Institute of Mechanics, Chinese Academy of Sciences for providing the test facility and Prof. F. Gao for the helpful discussions. 


\section{References}

Bourguet, R., Karniadakis G.E., Triantafyllou, M.S., 2015. On the validity of the independence principle applied to the vortex-induced vibrations of a flexible cylinder inclined at $60^{\circ}$. Journal of Fluids and Structures 53, 58-69.

Brika, D., Lanevill, A., 1993. Vortex-induced vibrations of a long flexible circular cylinder. Journal of Fluid Mechanics 250, 481-508.

Chong, M.S., Perry, A.E., Cantwell, B.J., 1990. A general classification of three-dimensional flow field. Physics of Fluids A(2), 765-777.

Det Norske Veritas. 2006. Free spanning pipelines. Recommended practice DNV-RP-F105.

Feng, C.C., 1968. The measurements of vortex-induced effects in flow past stationary and oscillating circular and D-section cylinder (Master's Thesis). University of British Columbia, Canada.

Franzini,G.R., Fujarra, A.L.C., Meneghini, J.R., Korkischko, I., Franciss, R., 2009. Experimental investigation of vortex-induced vibration on rigid, smooth and inclined cylinders. Journal of Fluids and Structures 25, 742-750.

Franzini, G.R., Gonçalves, R.T., Meneghini, J.R., Fujarra, A.L.C., 2013. One and two degrees-of-freedom vortex-induced vibration experiments with yawed cylinders. Journal of Fluids and Structures 42, 401-420.

Fredsøe, J., Sumer, B.M., Andersen J., Hansen, E.A., 1987. Transverse vibrations of a cylinder very close to a plane wall. Journal of Offshore Mechanics and Arctic Engineering 109, 52-60.

Govardhan, R.N., Williamson, C.H.K., 2006. Defining the 'modified Griffin plot' in vortex-induced vibration: revealing the effect of Reynolds number using controlled damping. Journal of Fluid Mechanics 561, 147-180.

Khalak, A., Williamson, C.H.K., 1999. Motions, forces and mode transitions in vortex-induced vibrations at low mass-damping. Journal of Fluids and Structures 13, 813-851.

Klamo, J.T., Leonard, A., Roshko, A., 2006. The effects of damping on the amplitude and frequency response of a freely vibrating cylinder in cross-flow. Journal of Fluids and Structures 22, 845856.

Jain, A., Modarres-Sadeghi, Y., 2013. Vortex-induced vibrations of a flexibly-mounted inclined cylinder. Journal of Fluids and Structures 43, 28-40. 
Lam, K., Lin, Y.F., Zou, L., Liu, Y., 2010. Investigation of turbulent flow past a yawed wavy cylinder. Journal of Fluids and Structures 26, 1078-1097.

Lei, C., Cheng, L., Kavanagh, K., 1999. Re-examination of the effect of a plane boundary on force and vortex shedding of a circular cylinder. Journal of Wind Engineering and Industrial Aerodynamics 80, 263-286.

Lucor, D., Karniadakis, G.E., 2003. Effects of oblique inflow in vortex-induced vibrations. Flow, Turbulence and Combustion 71, 375-389.

Prasanth, T.K., Premchandran, V., Mittal, S., 2011. Hysteresis in vortex-induced vibrations: critical blockage and effect of $m *$. Journal of Fluid Mechanics 671, 207-225.

Price, S.J., Sumner, D., Smith, J.D., Leong, K., Paigoussis, M.P., 2002. Flow visualization around a circular cylinder near to a plane wall. Journal of Fluids and Structures 16, 175-191.

Raghavan, K., Bernitsas, M.M., Maroulis, D.E., 2009. Effect of Bottom Boundary on VIV for Energy Harnessing at $8 \times 10^{3}<\operatorname{Re}<1.5 \times 10^{5}$. Journal of Offshore Mechanics and Arctic Engineering 131, 031102.

Ramberg, S., 1983. The effects of yaw and finite length upon vortex wakes of stationary and vibrating circular cylinders. Journal of Fluid Mechanics 128, 81-107.

Sarpkaya, T., 2004. A critical review of the intrinsic nature of vortex-induced vibrations. Journal of Fluids and Structures 19, 389-447.

Scarano, F., Riethmuller, M.L., 1999. Iterative multigrid approach in PIV image processing with discrete window offset. Experiments in Fluids 26, 513-523.

Singh, S.P., Mittal, S., 2005. Vortex-induced oscillations at low Reynolds numbers: Hysteresis and vortex-shedding modes. Journal of Fluids and Structures 20, 1085-1104.

Sumer, B.M., Fredsøe, J., 2006. Hydrodynamics around cylindrical structures. World Scientific Publishing, Singapore.

Tham, D.M.Y., Gurugubelli, P.S., Li,Z., Jaiman, R.K., 2015. Freely vibrating circular cylinder in the vicinity of a stationary wall. Journal of Fluids and Structures 59, 103-128.

Thapa, J., Zhao M., Zhou, T., Cheng, L., 2014. Three-dimensional simulation of vortex shedding flow in the wake of a yawed circular cylinder near a plane boundary at a Reynolds number of 500. Ocean Engineering 87, 25-39.

Tsahalis, D.T., 1983. The effect of sea-bottom proximity of the vortex-induced vibrations and 
fatigue life of offshore pipelines. Journal of Energy Resources Technology 105, 464-468.

Vandiver, J.K., 2012. Damping parameters for flow-induced vibration. Journal of Fluids and Structures 35, 105-119.

Wang, X.K., Hao, Z., Tan, S.K., 2013. Vortex-induced vibrations of a neutrally buoyant circular cylinder near a plane wall. Journal of Fluids and Structures 39, 188-204.

Wang, X.K., Tan, S.K., 2008. Near-wake flow characteristics of a circular cylinder close to a wall. Journal of Fluids and Structures 24, 605-627.

Williamson, C.H.K., Govardhan, R., 2004. Vortex-induced vibrations. Annual Review of Fluid Mechanics 36, 413-455.

Wu, X., Ge, F., Hong, Y., 2012. A review of recent studies on vortex-induced vibrations of long slender cylinders. Journal of Fluids and Structures 28, 292-308.

Yamamoto, T., Nath, J.H., Slotta, L.S., 1974. Wave Forces on Cylinders Near Plane Boundary. ASCE, Vol. 100, No. WW4.

Yang, B., Gao, F.P., Jeng, D.S., Wu, Y.X., 2009. Experimental study of vortex-induced vibrations of a cylinder near a rigid plane boundary in steady flow. Acta Mech Sina 25, 51-63.

Yang, B., Gao, F.P., Wu, Y.X., Li, D.H., 2006. Experimental study on vortex-induced vibrations of submarine pipeline near seabed boundary in ocean currents. China Ocean Engineering 20, $113-121$.

Zang, Z.P., Gao F.P., 2014. Steady current induced vibration of near-bed piggyback pipelines: configuration effects on VIV suppression. Applied Ocean Research 46, 62-69.

Zang, Z.P., Gao, F.P., Cui, J.S. 2013. Physical modeling and swirling strength analysis of vortex shedding from near-bed piggyback pipelines. Applied Ocean Research 40, 50-59.

Zhao, M., Cheng L., 2011. Numerical simulation of two-degree-of-freedom vortex-induced vibration of a circular cylinder close to a plane boundary. Journal of Fluids and Structures 27, 1097-1110.

Zhao, M., Cheng, L., Zhou, T., 2009. Direct numerical simulation of three-dimensional flow past a yawed circular cylinder of infinite length. Journal of Fluids and Structures 25, 831-847.

Zhou J., Adrian R.J., Balachandar S., Kendal T.M. 1999. Mechanisms for generating coherent packets of haripin vortices in channel flow. Journal of Fluid Mechanics 387: 353-359.

Zhou, T., Razali. S.F.M., Hao, Z., Cheng, L. 2011. On the study of vortex-induced vibration of a 
cylinder with helical strakes. Journal of Fluids and Structures 27: 903-917.

Zhou, T., Wang, H., Razali, S.F.M., Zhou, Y., Cheng, L., 2010. Three-dimensional vorticity measurements in the wake of a yawed circular cylinder, Physics of Fluids 22, 015108. 
Figures 

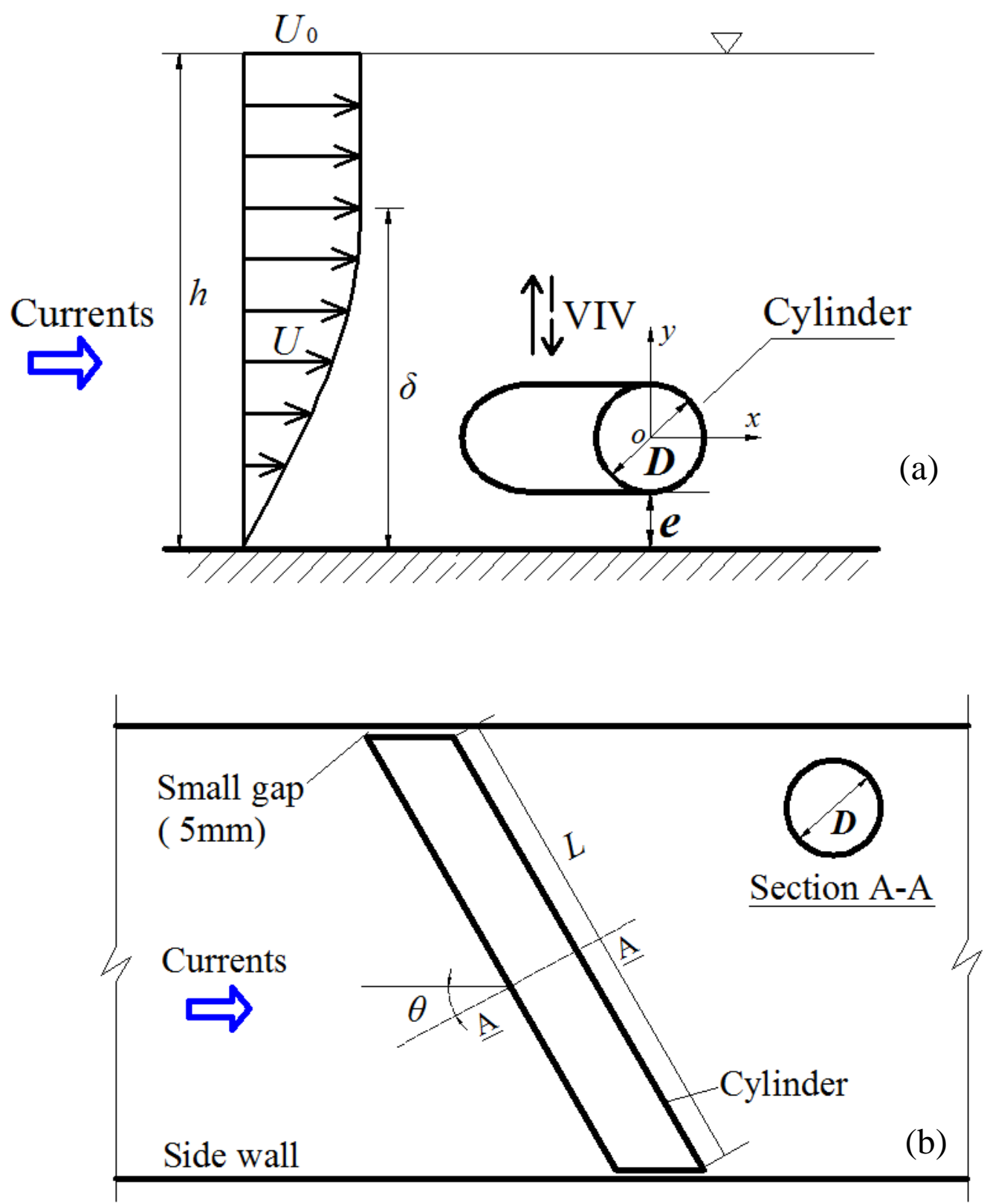

Fig. 1. Sketches of cylinder arrangements near a wall under an oblique angle. (a) Side view. (b) Top view. 


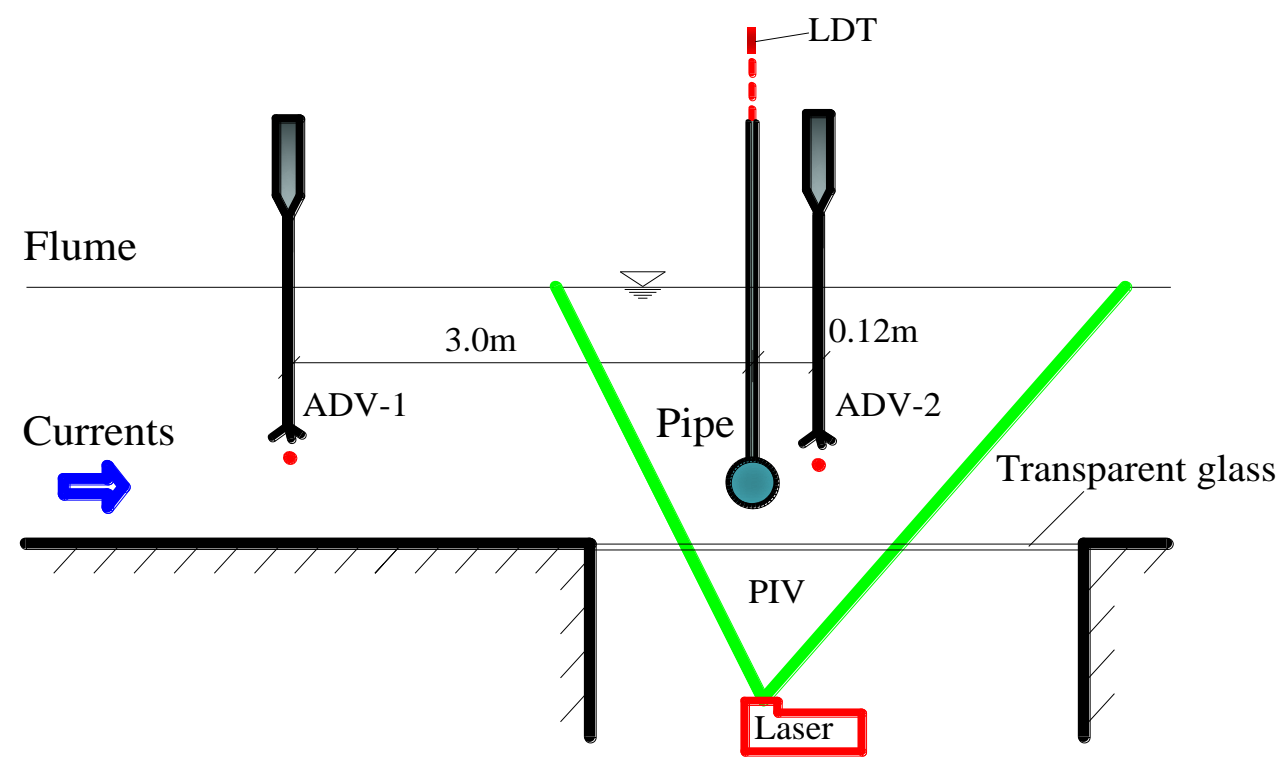

Fig. 2. Sketch of synchronous measurements of fluid-structure interactions. 


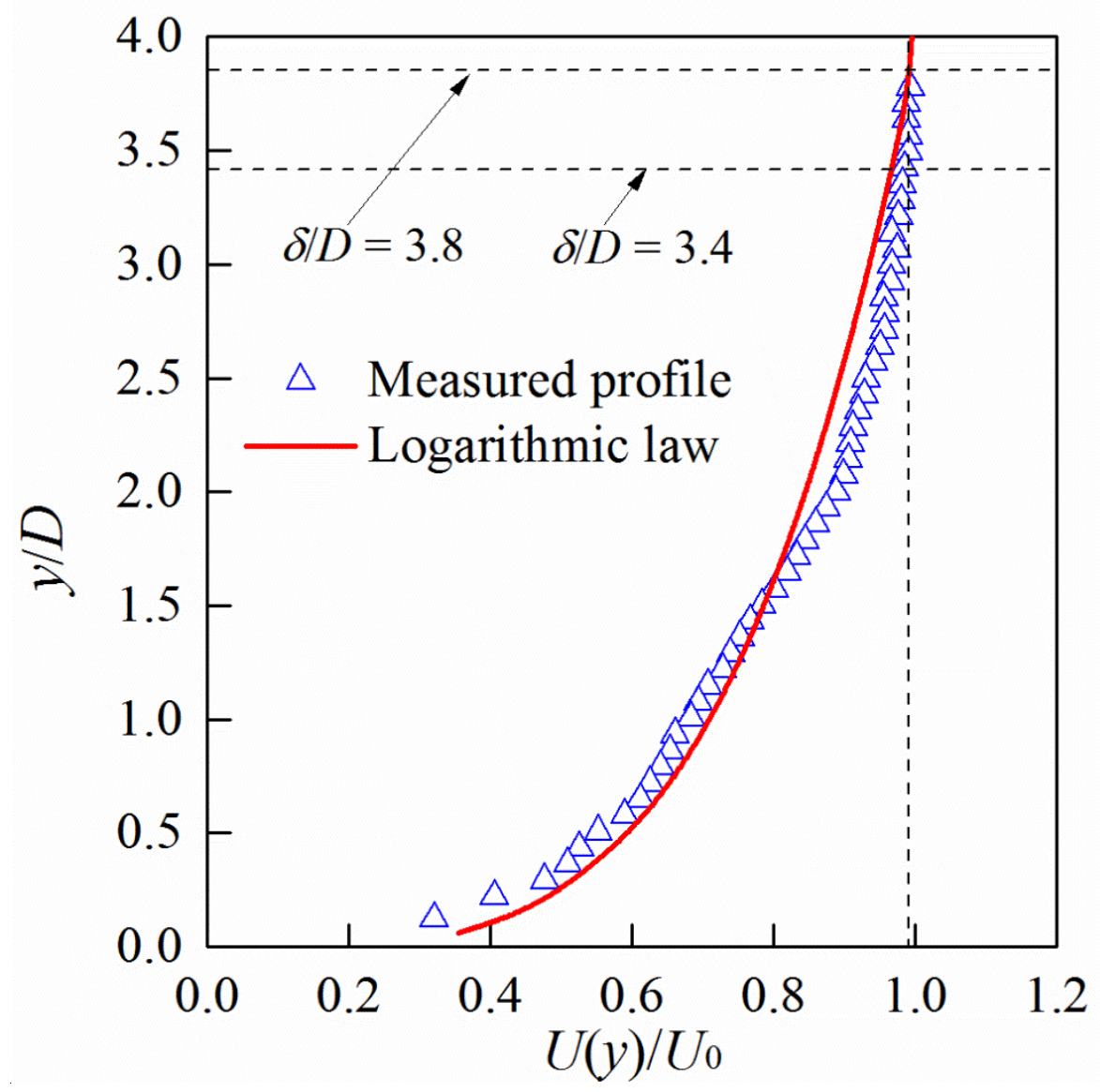

Fig. 3. Measured mean velocity distribution in the boundary layer of the test section and comparison with the prediction using the Logarithmic law. The vertical dashed line represents $U(y) / U_{0}=0.99$ and the corresponding boundary layer thicknesses are $\delta / D=3.4$ (from the measured data) and 3.8 (from the Logarithmic law). 


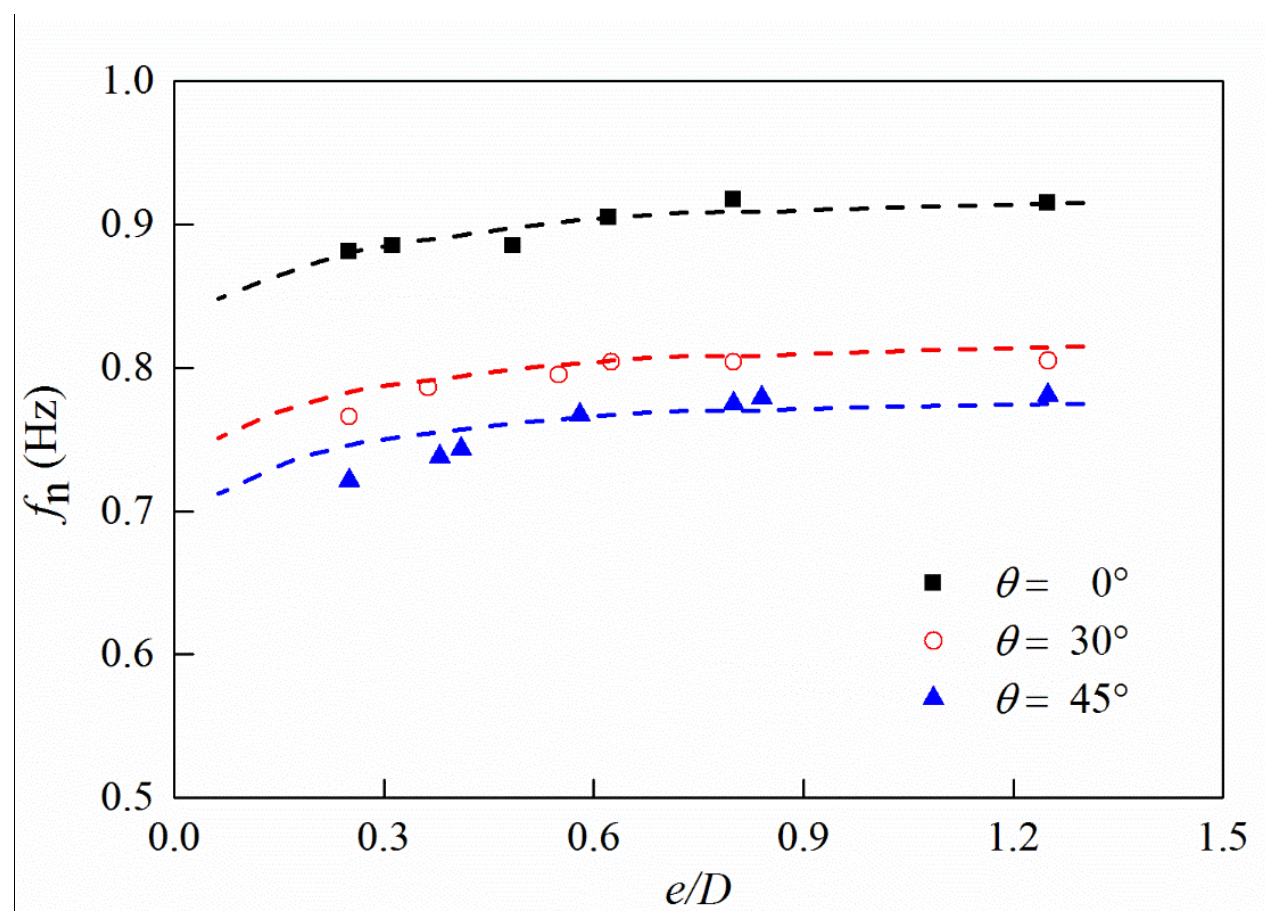

Fig. 4. Variations of the natural frequency with gap ratio for different oblique angles. The dashed lines represent the predicted values for different angles using Eq. (4). 

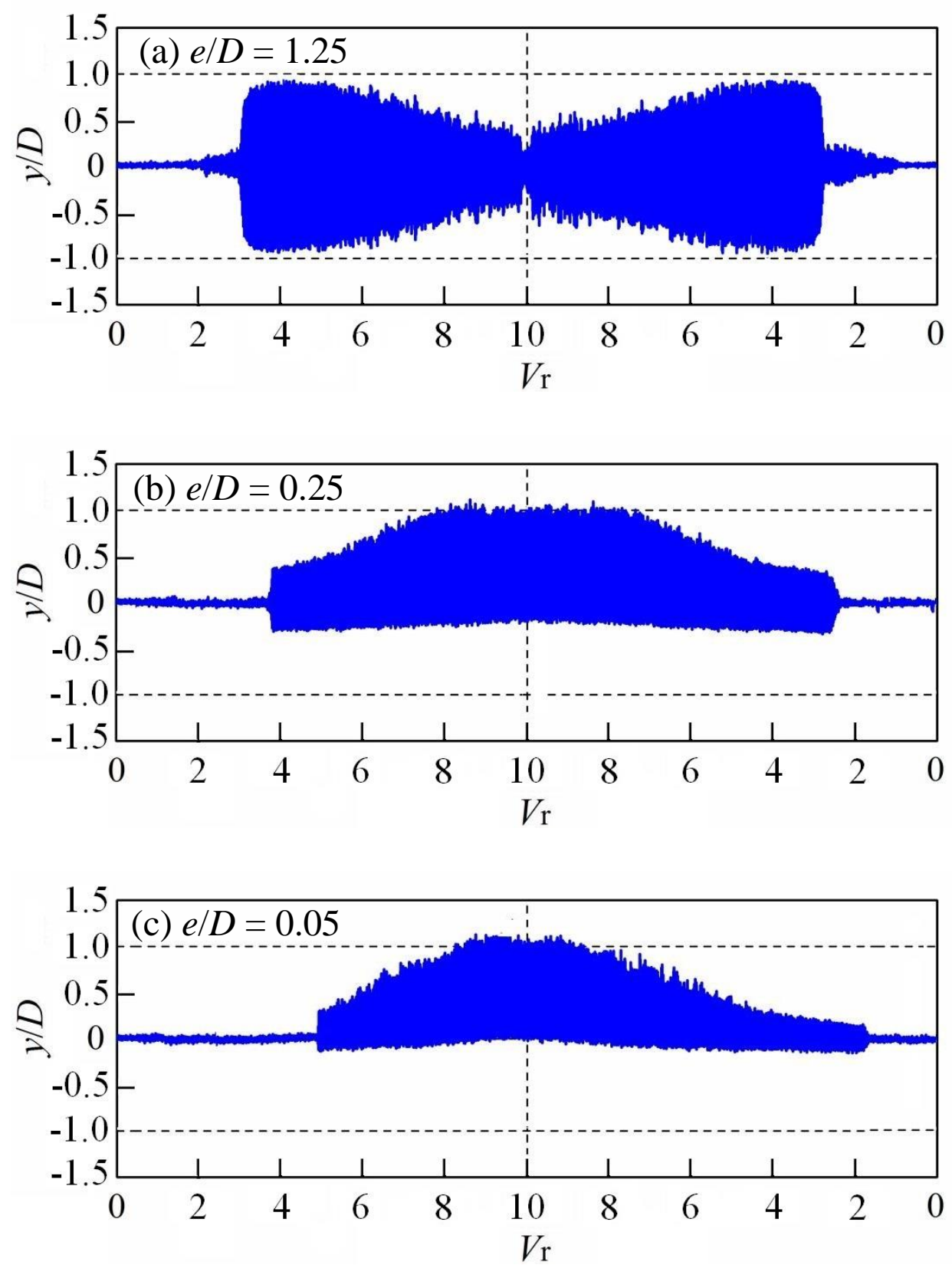

Fig. 5. Cylinder displacements varying with reduced velocity for $\theta=0^{\circ}$ at different gap ratios both showing increasing and decreasing velocities. (a) $e / D=1.25$; (b) $e / D=0.25$; (c) $e / D=0.05$. 

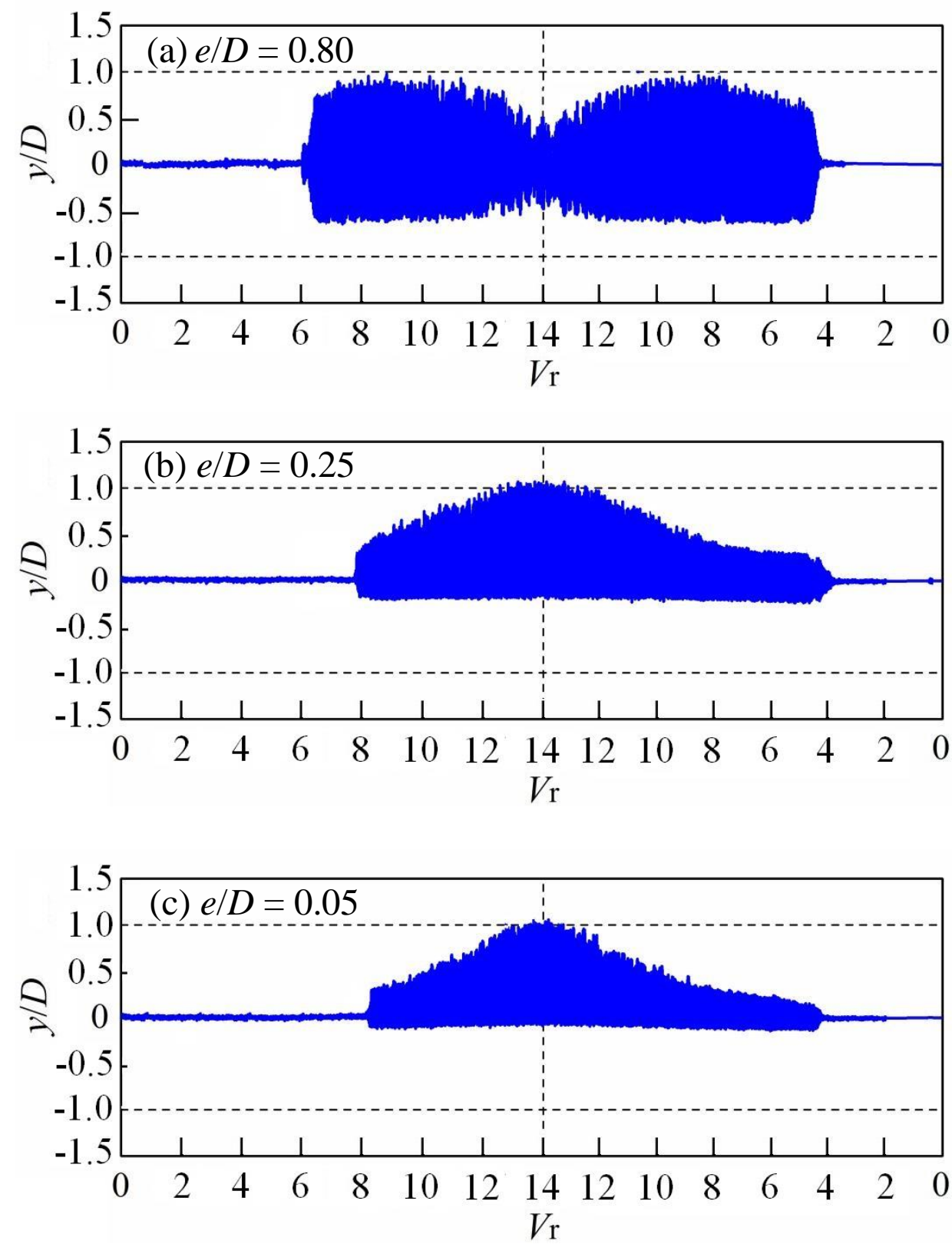

Fig. 6. Cylinder displacements varying with reduced velocity for $\theta=45^{\circ}$ at different gap ratios both showing increasing and decreasing velocities. (a) $e / D=0.80$; (b) $e / D=0.25$; (c) $e / D=0.05$. 

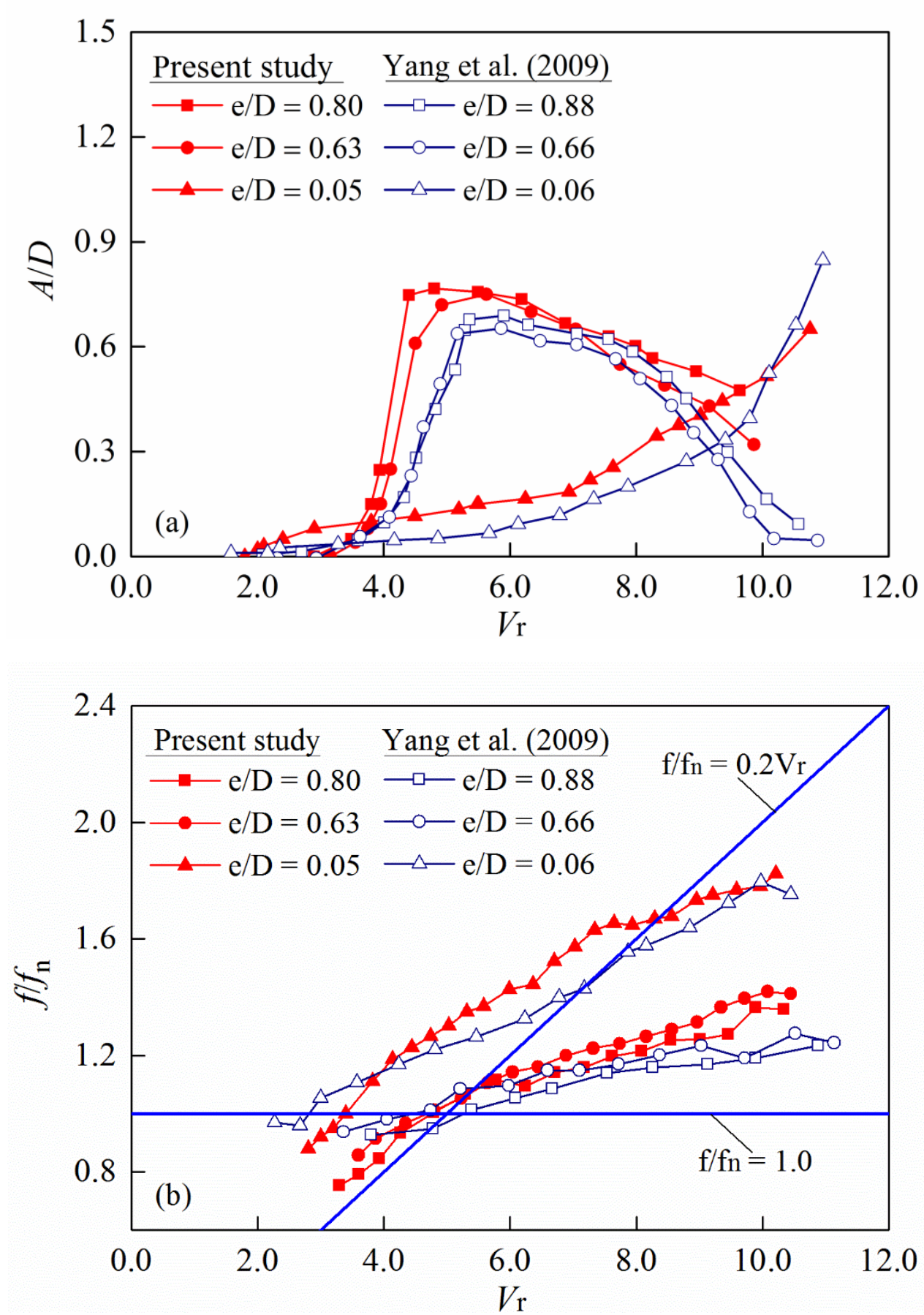

Fig. 7. Comparisons of the vibration amplitude $A / D$ and the frequency ratio $f / f n$ between the present results and those reported by Yang et al. (2009) in cross flows $\left(\theta=0^{\circ}\right)$. (a) Amplitude; (b) Frequency. 

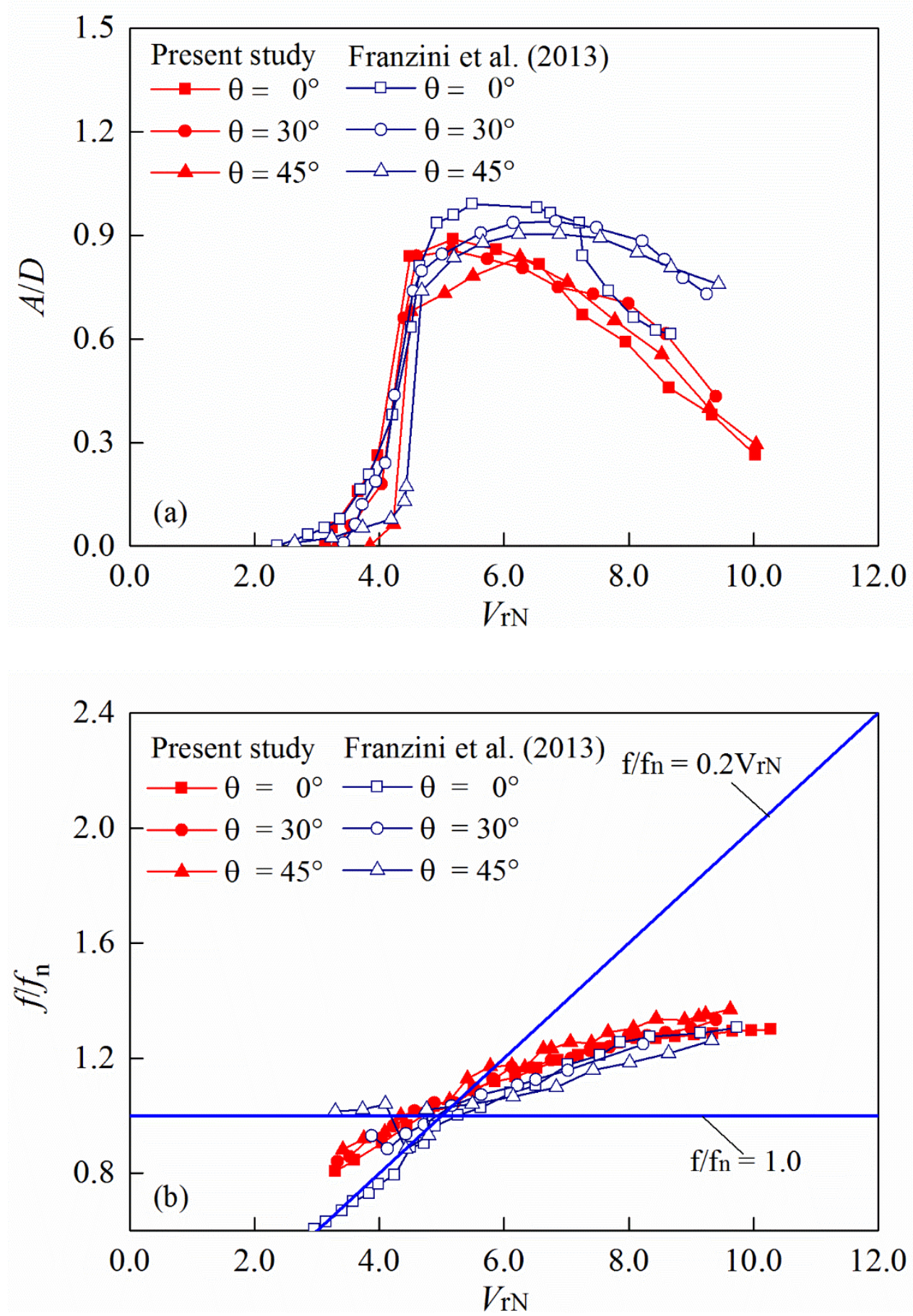

Fig. 8. Comparisons of the vibration amplitude $A / D$ and the frequency ratio $f / f n$ between the present test results and those of Franzini et al (2013) for VIV of a wall-free cylinder $(e / D \approx \infty)$ in oblique flows. (a) Vibration amplitude $A / D$; (b) Frequency ratio $f / f_{\mathrm{n}}$. 


\section{Increasing velocity}
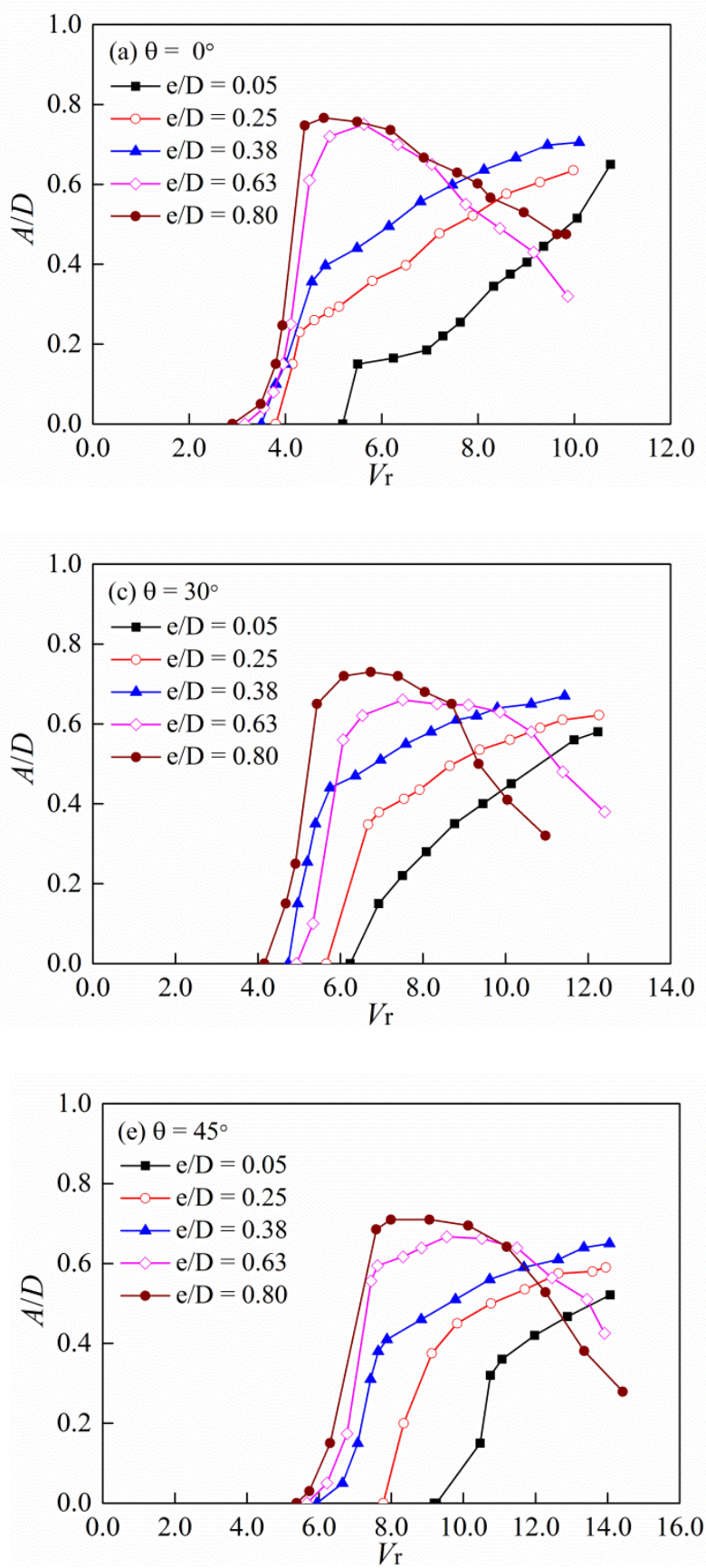

Decreasing velocity
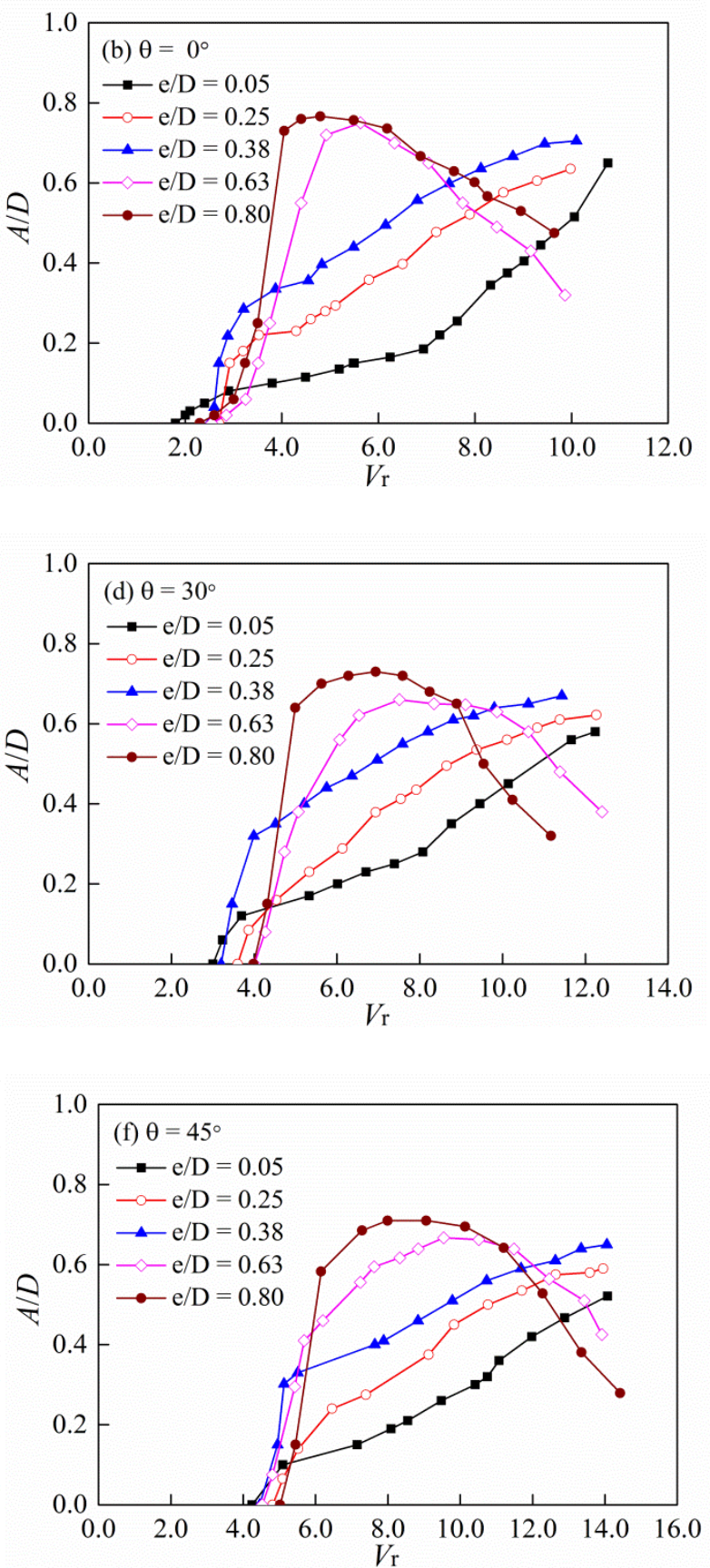

Fig. 9. Variation of $A / D$ with $V_{\mathrm{r}}$ for different values of $e / D$ at different oblique angles both for increasing and decreasing velocities. The left column is for increasing velocity and the right column is for decreasing velocity. (a) and (b) $\theta=0^{\circ}$; (c) and (d) $\theta=30^{\circ}$; (e) and (f) $\theta=45^{\circ}$. 

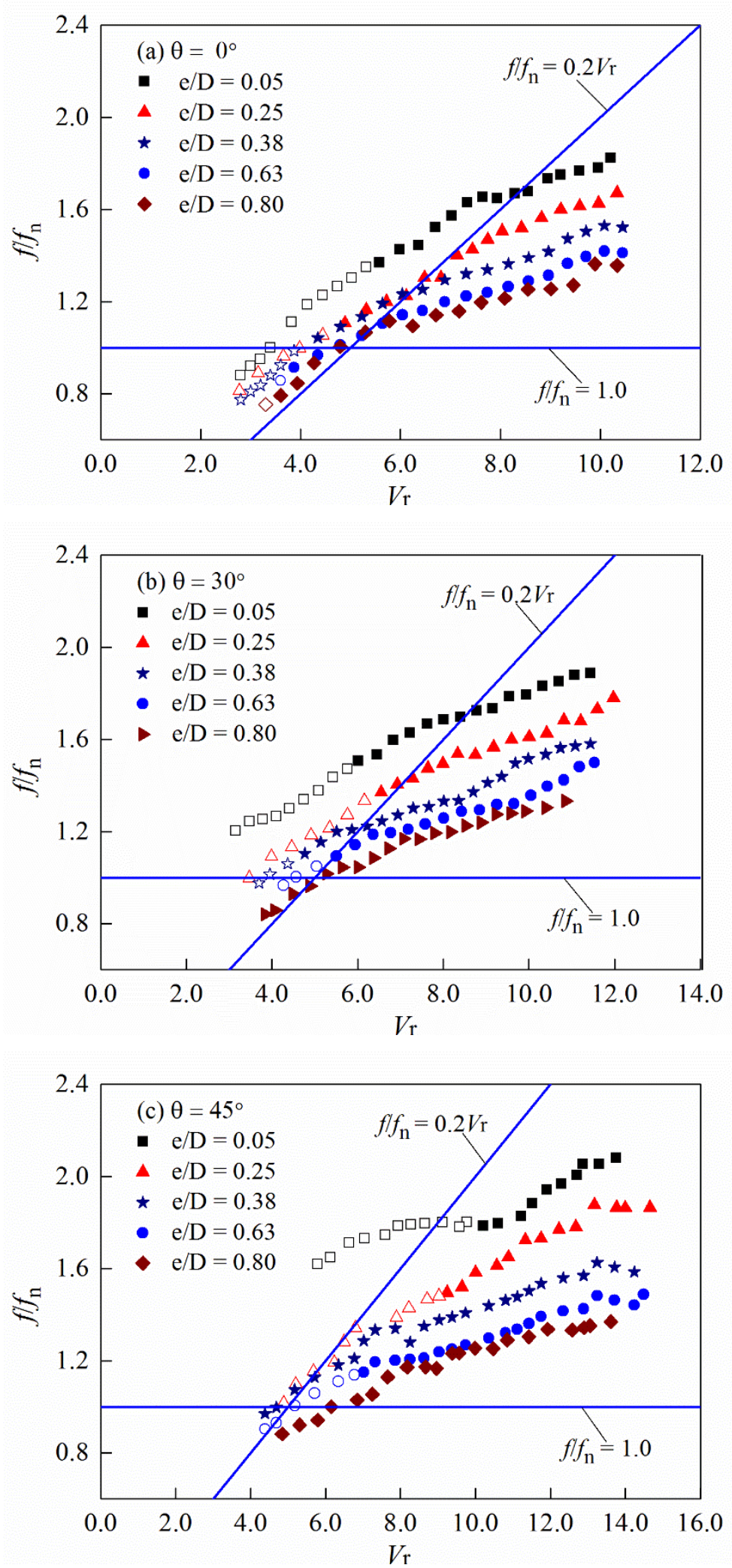

Fig. 10. Variations of $f / f_{\mathrm{n}}$ with $V_{\mathrm{r}}$ for different values of $e / D$ at different oblique angles. (a) $\theta=0^{\circ}$; (b) $\theta=30^{\circ}$; (c) $\theta=45^{\circ}$. The hollow symbols are for decreasing velocity only, and the solid symbols are for both increasing and decreasing velocities. 


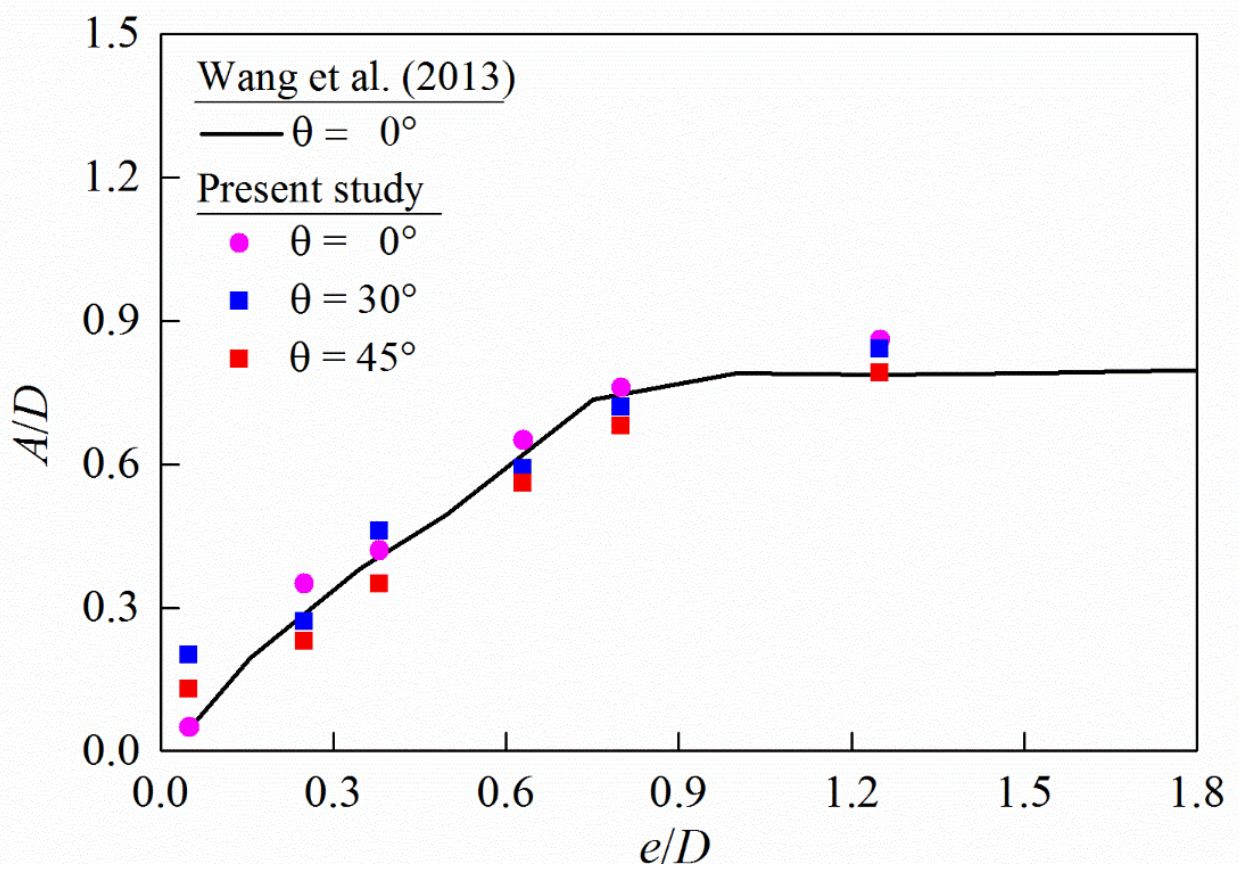

Fig. 11. Variation of $A / D$ with $e / D$ at $V_{\mathrm{rN}}=5.1$ and $R e \approx 3.0 \times 10^{4}$ for different oblique angles and comparison with results reported by Wang et al. (2013). 
Increasing velocity
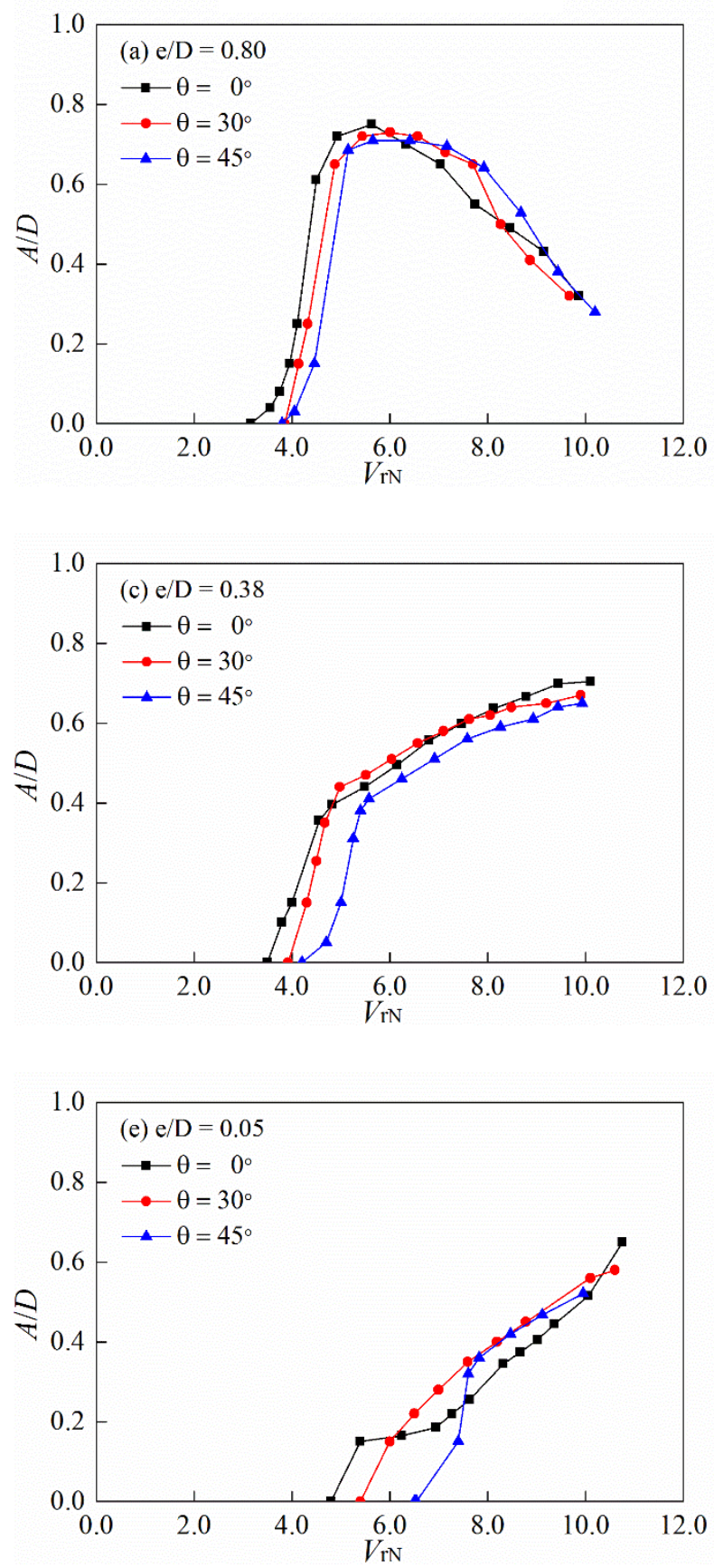

Decreasing velocity
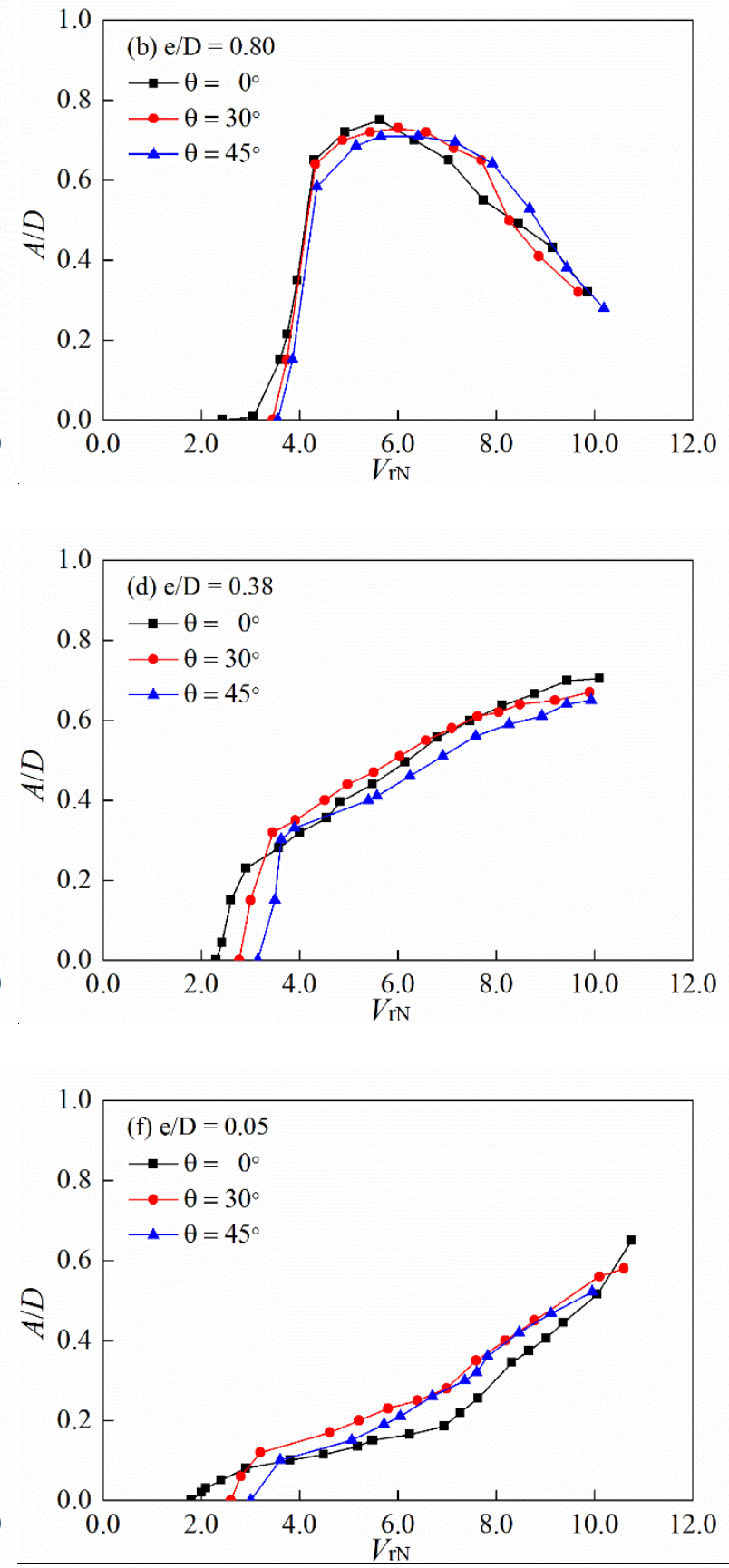

Fig. 12. Variations of $A / D$ with $V_{\mathrm{rN}}$ for different oblique angles and gap ratios. The left column is for increasing velocity and the right column is for decreasing velocity. (a) and (b) $e / D=0.80$; (c) and (d) $e / D=0.38 ;$ (e) and (f) $e / D=0.05$. 

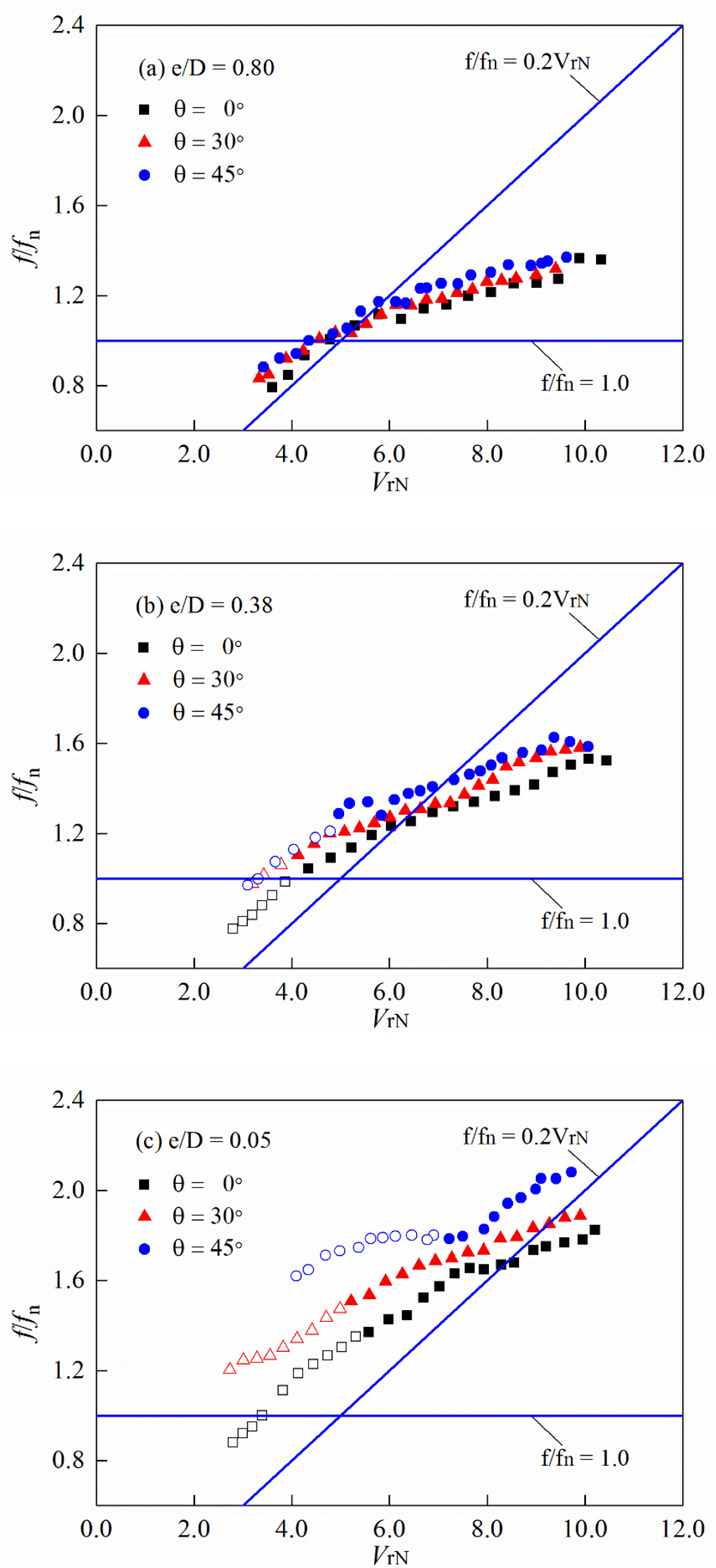

Fig. 13. Variations of $f l f_{\mathrm{n}}$ with $V_{\mathrm{rN}}$ for different oblique angles and cylinder gap ratios. (a) $e / D=0.80$; (b) $e / D=0.38$; (c) $e / D=0.05$. The hollow symbols are for decreasing velocity only, and the solid symbols are for both increasing and decreasing velocities. 


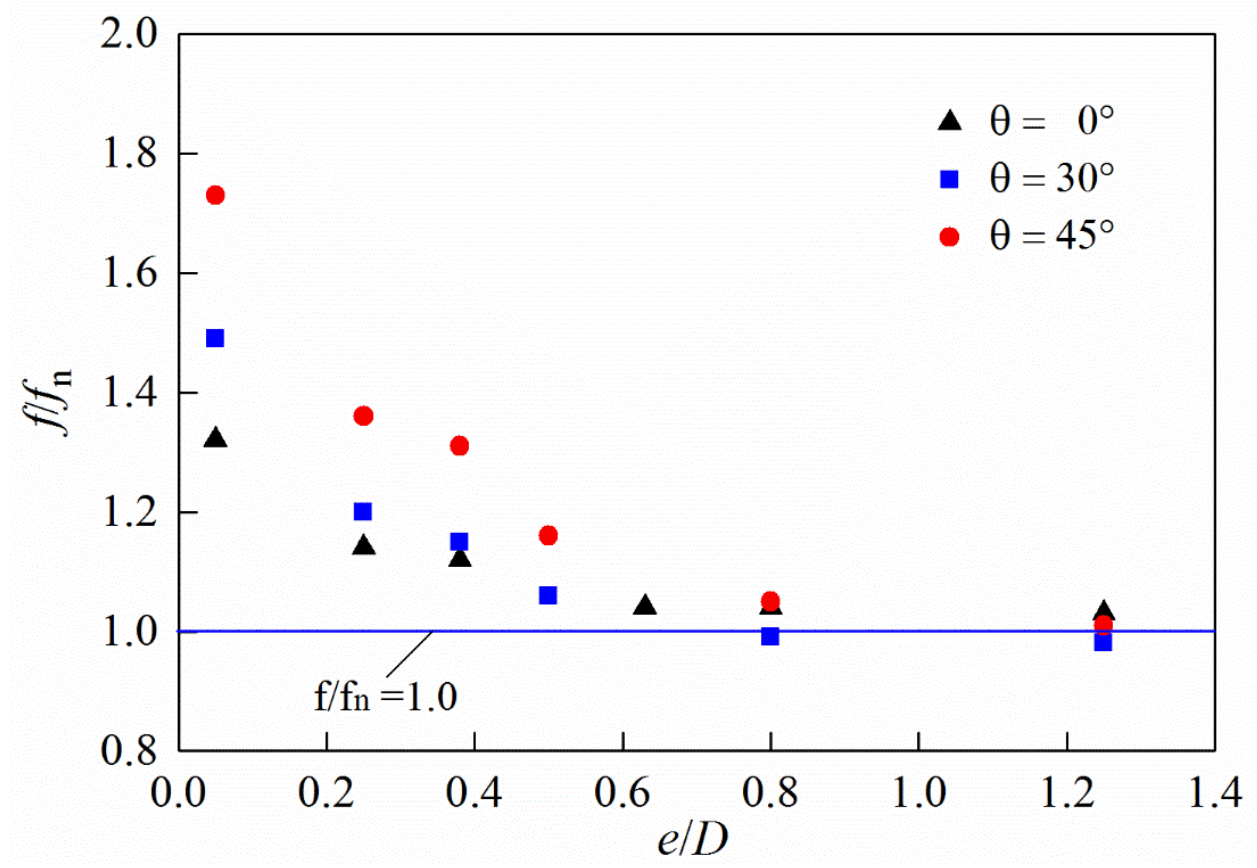

Fig. 14. Variations of $f / f_{\mathrm{n}}$ with $e / D$ for different oblique angles at $V_{\mathrm{rN}}=5.1$. 

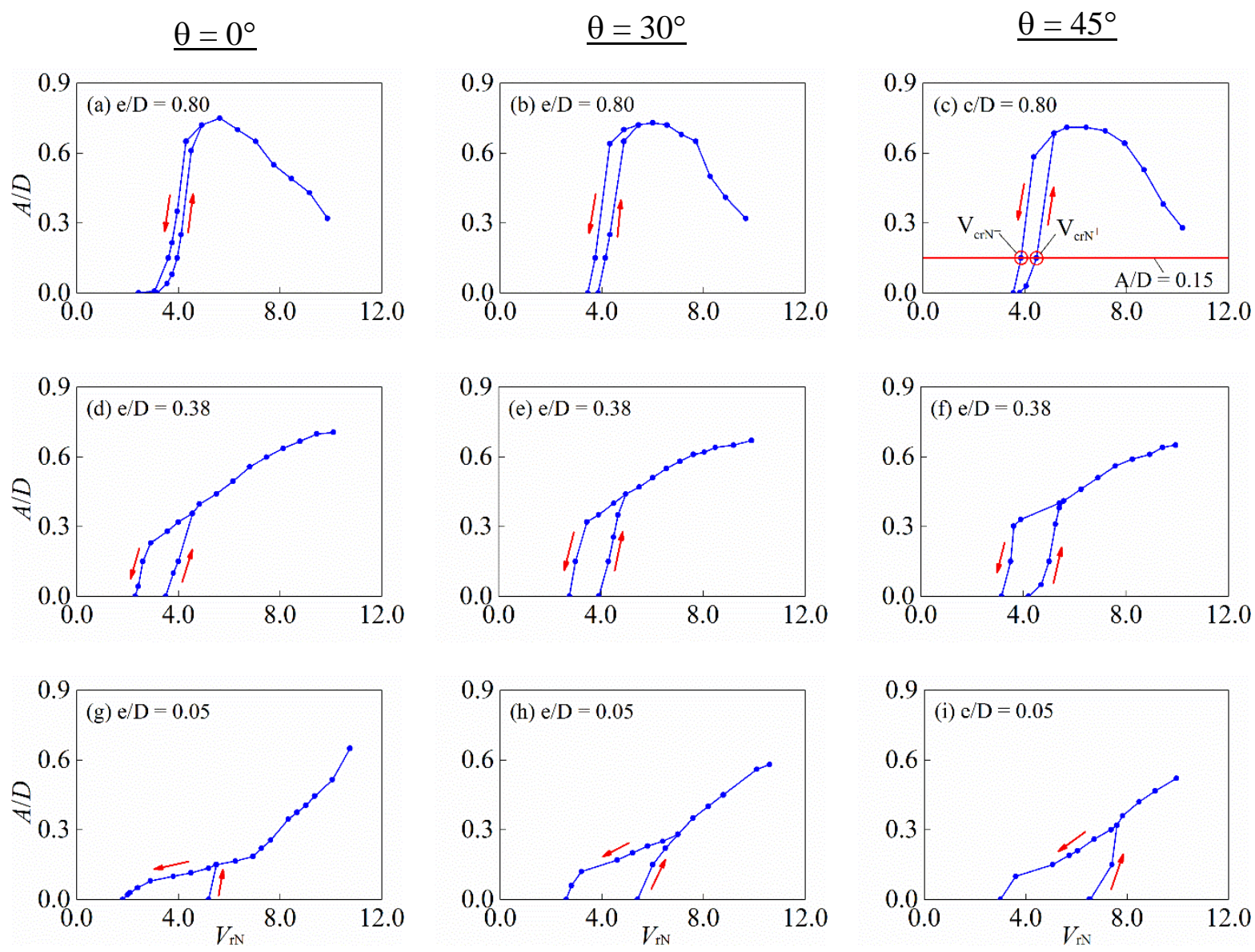

Fig. 15. Onset of VIV for various values of $e / D$ and $\theta$ with increasing and decreasing velocities. The left, middle and right columns are for $\theta=0^{\circ}, 30^{\circ}$ and $45^{\circ}$, respectively. (a) $\sim$ (c) $e / D=0.80$; (d) (f) $e / D=0.38 ;(\mathrm{g}) \sim(\mathrm{i}) e / D=0.05$. The arrows associated with the initial branch indicate the direction of the variation of amplitude with the increasing (or decreasing) velocity. The arrows pointing upward represent increasing velocity and those pointing downward represent decreasing velocity. The line at $A / D=0.15$ in (c) is for the definitions of the upper and lower critical normal reduced velocity, namely, $V_{\mathrm{crN}}{ }^{+}$and $V_{\mathrm{crN}}{ }^{-}$for the increasing and decreasing velocities. 

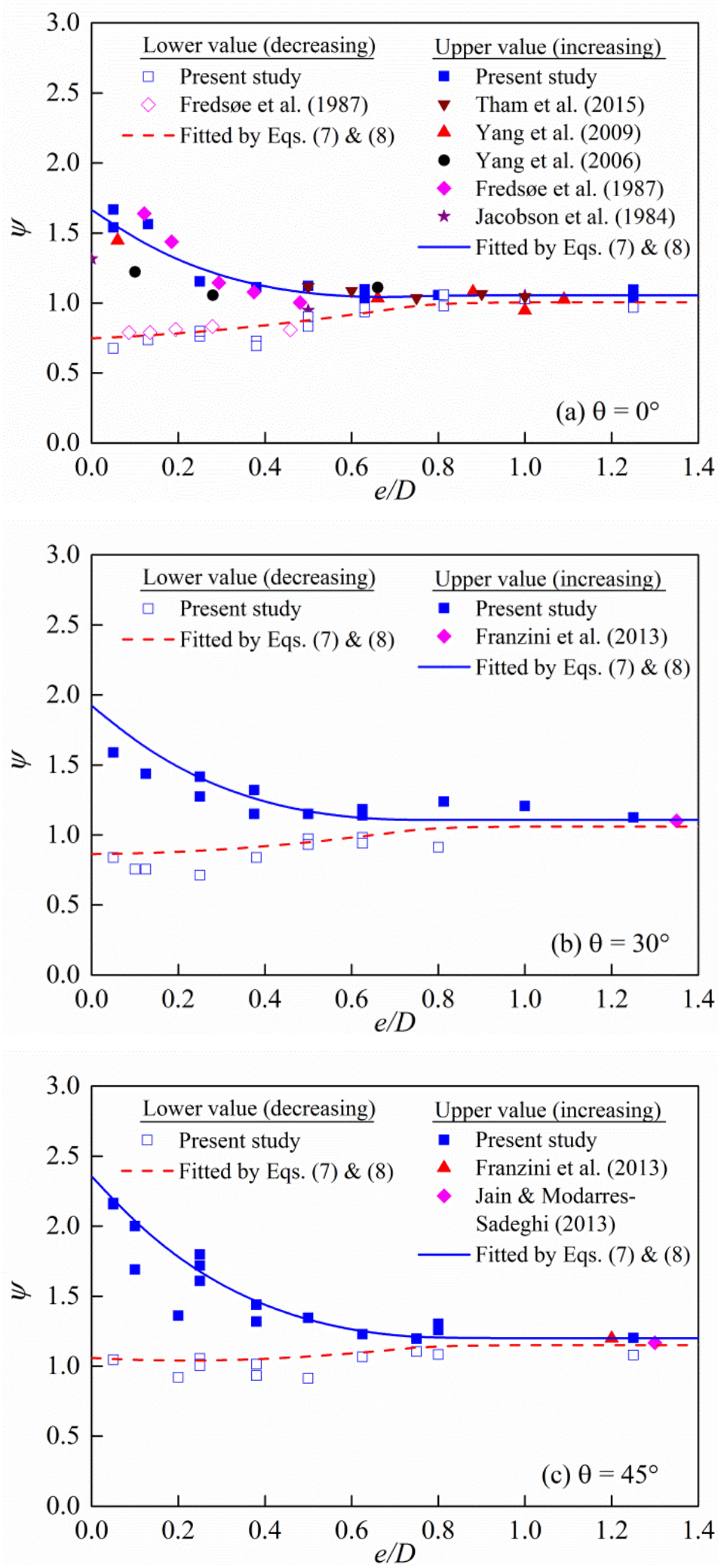

Fig. 16. Variation of $\psi$ with $e / D$ for different oblique angles and comparison with results reported previously. (a) $\theta=0^{\circ}$; (b) $\theta=30^{\circ}$; (c) $\theta=45^{\circ}$. Eq. (7) is use for fittings as $e / D \leq 0.8$ and Eq. (8) is used for fittings as $e / D>0.8$. 


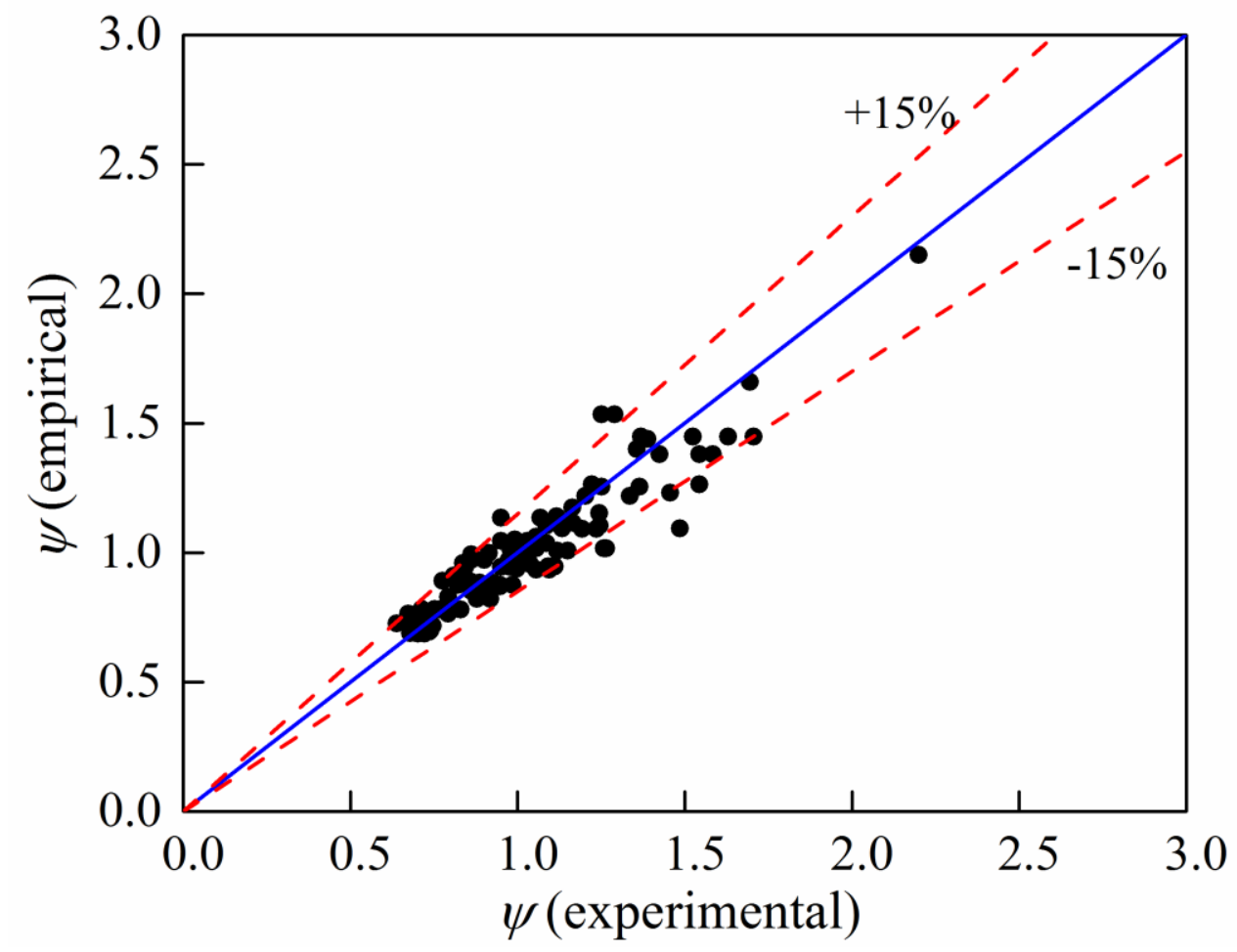

Fig. 17. Correlation between the experimental results of $\psi$ and those predicted by Eqs. (7) and (8). The solid blue line has a slope of 1 representing perfect correlation between the experimental and the predicted results of $\psi$. The dashed lines represent $\pm 15 \%$ deviation from the solid line. 


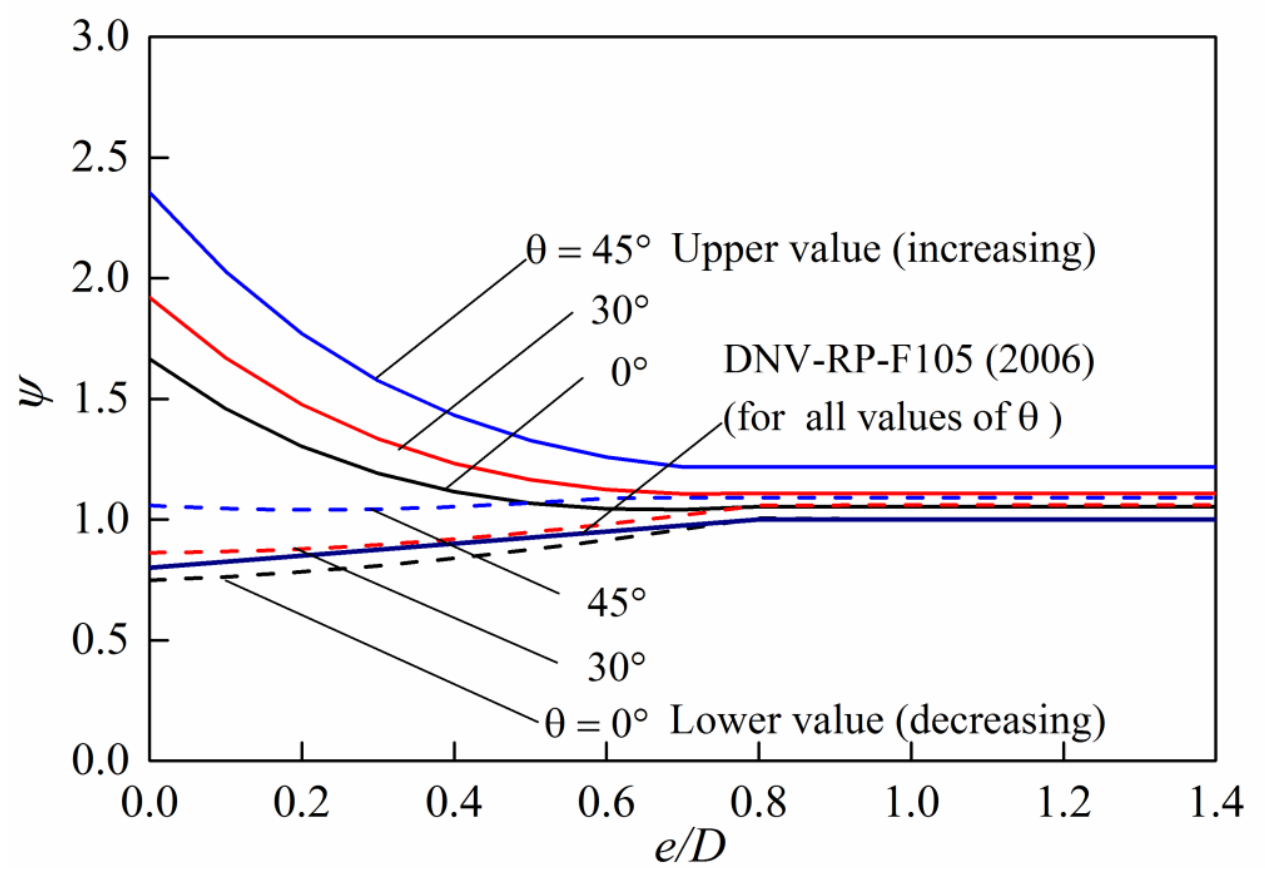

Fig. 18. Design plot for the correction factor $\psi$ under various flow conditions (for $\theta \leq 45^{\circ}$ ) with $\alpha=$ 0.098 and $R e=2.0 \sim 6.0 \times 10^{4}$. Solid curves represent increasing velocity while dashed curves represent decreasing velocity. The result by DNV-RP-F105 (2006) is also shown with a thick blue curve. Eq. (7) is used for fittings as $e / D \leq 0.8$ and Eq. (8) is used for fittings as $e / D>0.8$. 

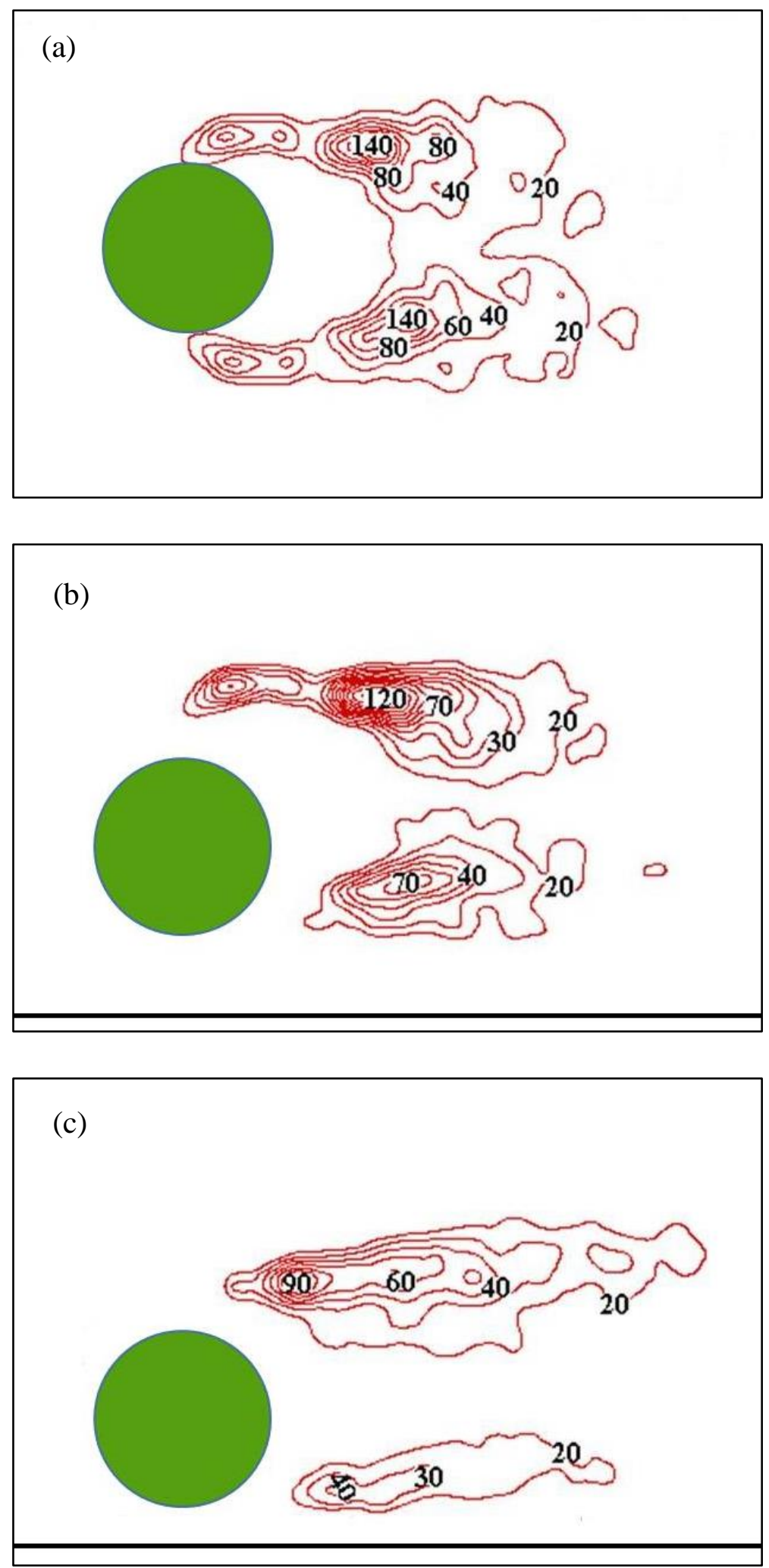

Fig. 19. Time-averaged swirling strength field (unit: $\mathrm{s}^{-2}$ ) of a stationary cylinder in a cross-flow ( $\theta$ $=0^{\circ}$ ) for different gap ratios at $R e=2.4 \times 10^{4}$. (a) $e / D \approx \infty$ (isolated cylinder); (b) $e / D=0.63$; (c) $e / D=0.25$. 

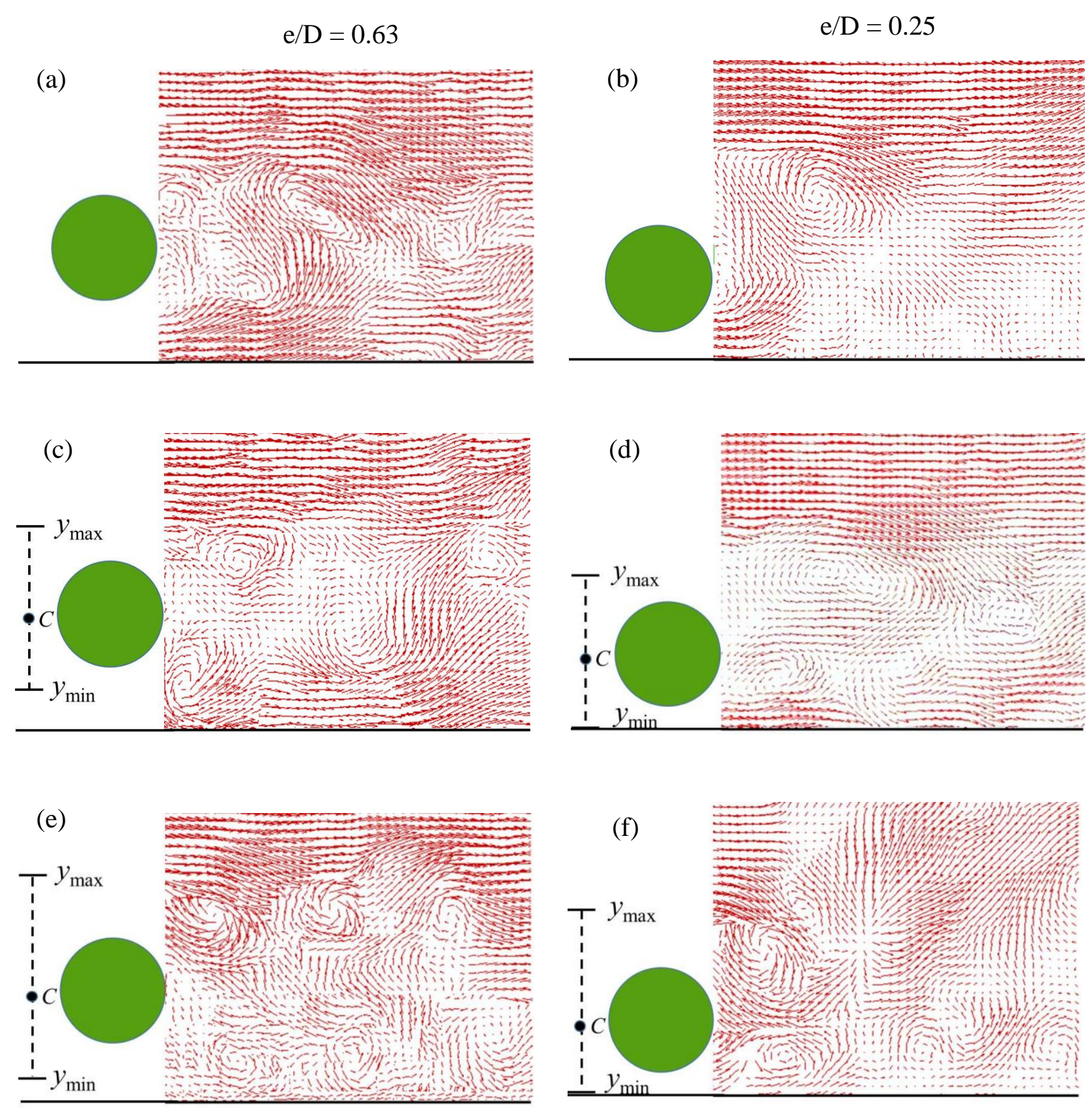

Fig. 20. Representative snapshots of the velocity vector around the cylinder in a cross-flow ( $\theta=$ $\left.0^{\circ}\right)$ near the wall for $V_{\mathrm{r}}=4.0$. The left column is for $e / D=0.63$ and the right column is for $e / D=$ 0.25. (a) and (b): stationary cylinder; (c) (d): vibrating cylinder with increasing velocity; (e) and (f): vibrating cylinder with decreasing velocity. The location and the maximum and minimum locations of the cylinder are also marked in the figures. The flow direction is from left to right. 